\title{
Universal aspects of neutron halos in light exotic nuclei
}

Tobias Frederico

Instituto Tecnológico de Aeronáutica

São José dos Campos - Brazil tobias@ita.br 


\section{Light-neutron rich nuclei}
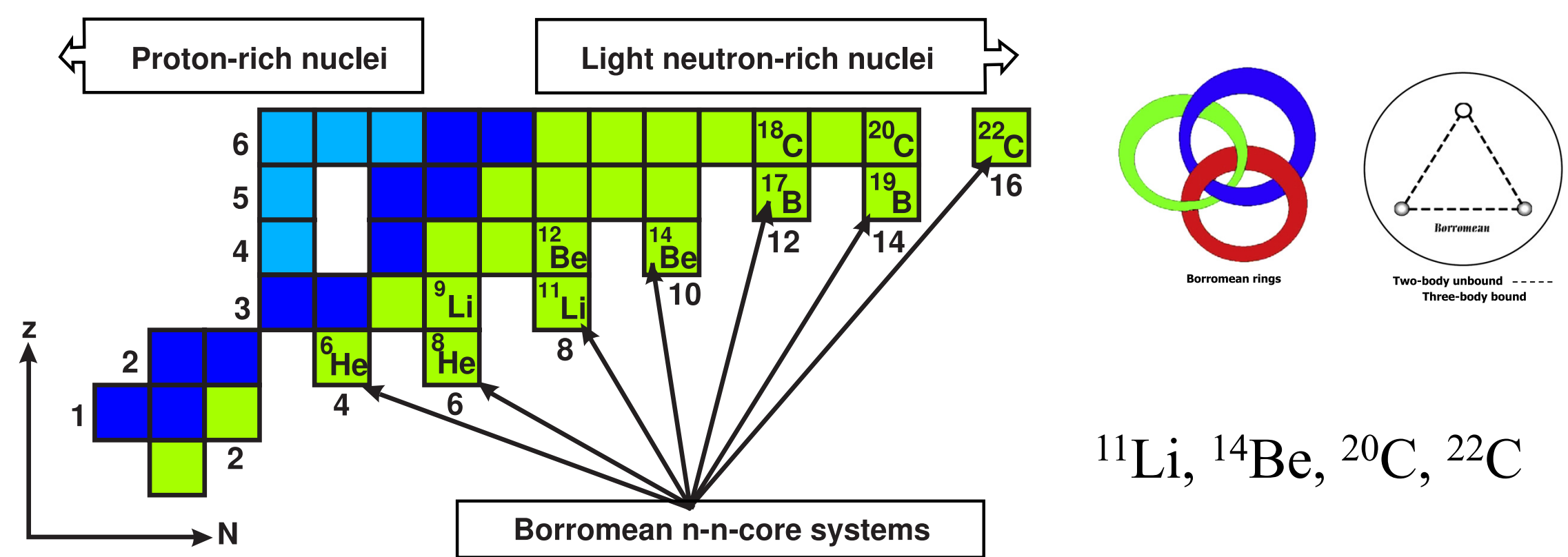

${ }^{11} \mathrm{Li},{ }^{14} \mathrm{Be},{ }^{20} \mathrm{C},{ }^{22} \mathrm{C}$

C.A. Bertulani, Nuclear Physics in a Nutshell, Princeton University Press, 2007.

TF, Delfino, Tomio, Yamashita, "Universal aspects of light halo nuclei

Prog. Part. Nucl. Phys. 67 (2012) 939”

Tanihata,Savajols Kanungo. "Recent experimental progress in nuclear halo structure studies Prog. Part. Nucl. Phys. 68 (2012) 215"

Zinner, Jensen. "Comparing and contrasting nuclei and cold atomic gases".

J. Phys. G: Nucl. Part. Phys. 40 (2013) 053101 


\section{Two-neutron weakly bound s-wave three-body halo nuclei}

$$
\mathbf{S}_{2 \mathbf{n}}=369 \mathrm{keV} \text { - Smith et al. PRL101, } 202501 \text { (2008) }
$$

Tanihata et al., PRL55, 2676 (1985)
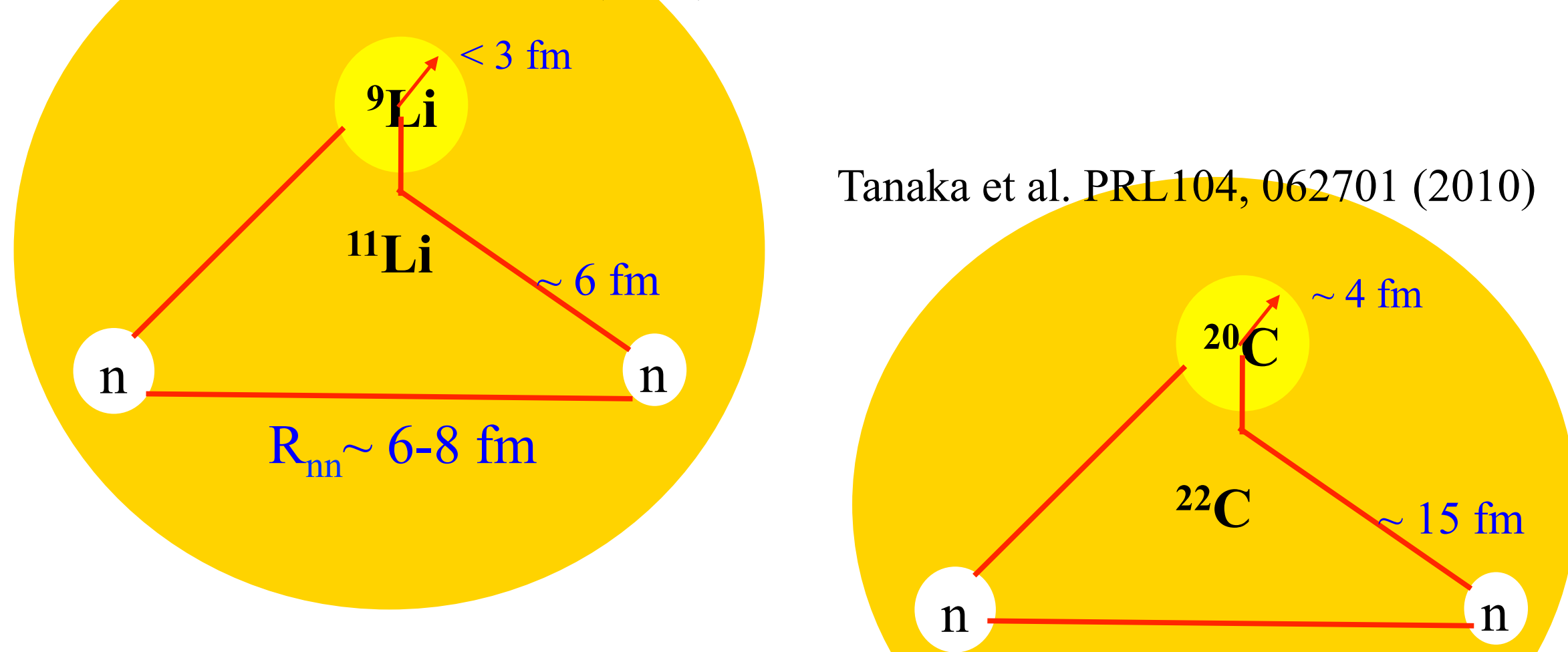

$\mathbf{S}_{\mathbf{2 n}}<70$ keV Mosby et al. NPA 909, 69 (2013) 
Weakly bound quantum systems

$$
\left(E-H_{0}\right) \psi=0
$$

- Almost everywhere the wf is an eigenstate of $\mathrm{H}_{0}$ - short-range force

- Physics: symmetry, scales and dimension (\& mass ratios)

$$
\rightarrow \text { Universality (model independence) }
$$

Generalization: "The few scales of nuclei and nuclear matter" Delfino, TF, Timóteo, Tomio. PLB 634 (2006) 185 
Two-body s-wave:

$$
\begin{array}{r}
k \cot (\delta)=-\frac{1}{a}+\frac{r_{0}}{2} k^{2}+\cdots \\
|a|>>r_{0}
\end{array}
$$

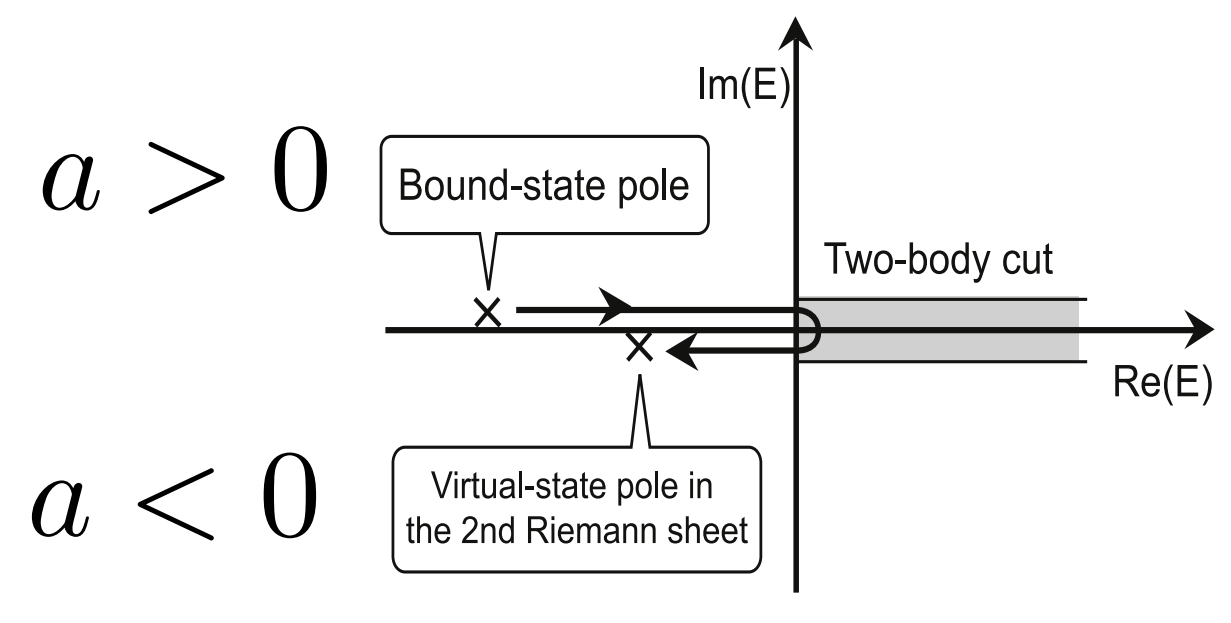

- ${ }^{1} \mathrm{~S}_{0}$ nn state $\mathrm{E}_{\text {virtual }}=-143 \mathrm{keV}(\mathrm{a}=-17 \mathrm{fm})$

- $\mathrm{S}$ n-core virtual $\left({ }^{10} \mathrm{Li} \sim-25 \mathrm{keV}\right)$ or bound $\left({ }^{19} \mathrm{C} \sim 500 \mathrm{keV}\right)$ 


\section{Three-boson}

Subtle three-body phenomenum in $\mathrm{L}=0$ :

\begin{tabular}{|c|c|}
\hline Thomas collapse (1935) & Efimov effect (1970) \\
\hline $\mathrm{r}_{\mathrm{o}} \rightarrow 0$ & $|\mathrm{a}| \rightarrow \infty$ \\
\hline Route to collapse? & $\begin{array}{l}\text { infinitely many bound states } \\
\text { condensing at } E=0\end{array}$ \\
\hline
\end{tabular}

Adhikari, Delfino,TF,Goldman,Tomio, PRA37 (1988) 3666

One three-body scale is necessary to represent short-range physics !!!! $\&$ discrete scaling

Jensen, Riisager, Fedorov, Garrido, RMP76, 215 (2004) Braaten, Hammer Phys. Rep.428, 259 (2006) 


\section{Efimov States - Bound and virtual states (3 identical bosons)}

Correlations between observables: Jensen, Fedorov,Yamashita, Hammer, Platter,

Gattobigio,Kievsky,Kolganova,Van Kolck,Bedaque,Phillips,...

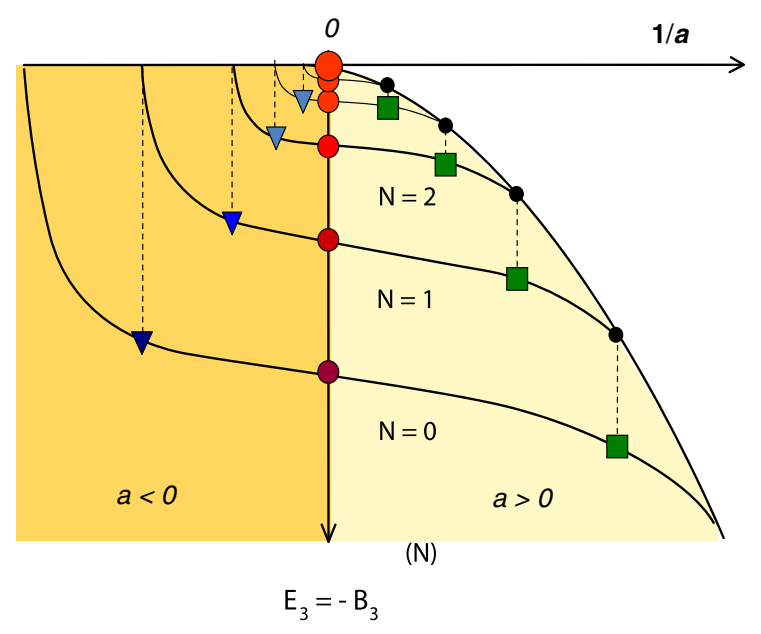

Scaling plot from zero range force

[1] Cornelius, Glöckle. JCP 85, 1 (1996).

[2] Huber. PRA31, 3981 (1985).

[3] Barletta, Kievsky. PR A64, 042514 (2001).

[4] Fedorov, Jensen. JPA34, 6003 (2001).

[5] Kolganova, Motovilov, Sofianos. PRA 56, R1686 (1997).

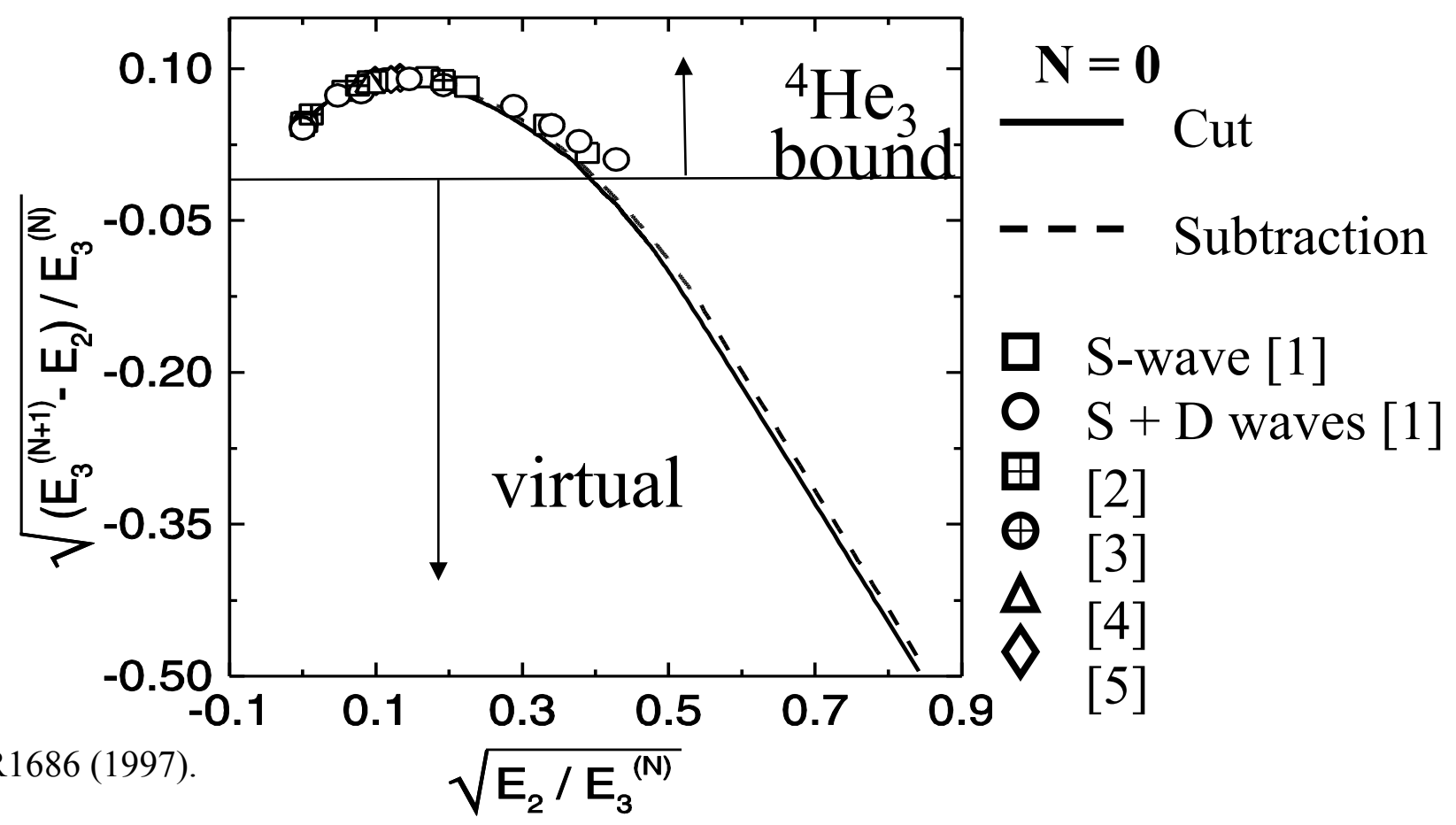

- Scaling limit: T. Frederico, LT, A. Delfino and E. A. Amorim, PRA60, R9 (1999)

- Limit cycle: Mohr et al Ann.Phys. 321 (2006)225

- Correlation between observables: Phillips Plot ${ }^{2} \mathrm{a}_{\text {nd }}$ v.s. $\mathrm{E}_{\text {triton }}$ 


\section{Four-bosons}

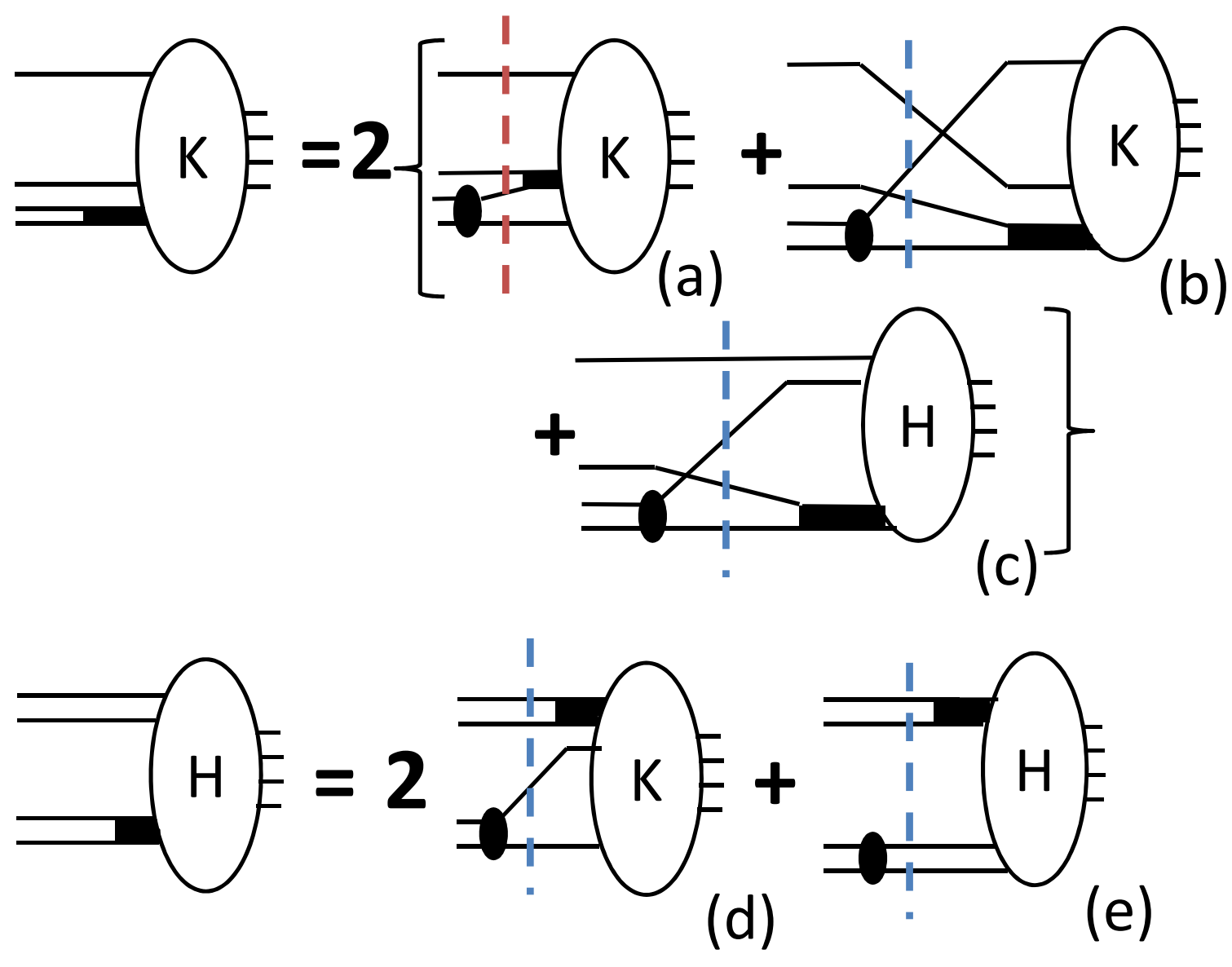

Subtracted Green's Functions: $G_{0}^{(N)}=\frac{1}{E-H_{0}}-\frac{1}{-\mu_{N}^{2}-H_{0}}$ with $\mu_{3}$ (RED): 3B scale \& $\mu_{4}$ (BLUE): 4B scale 

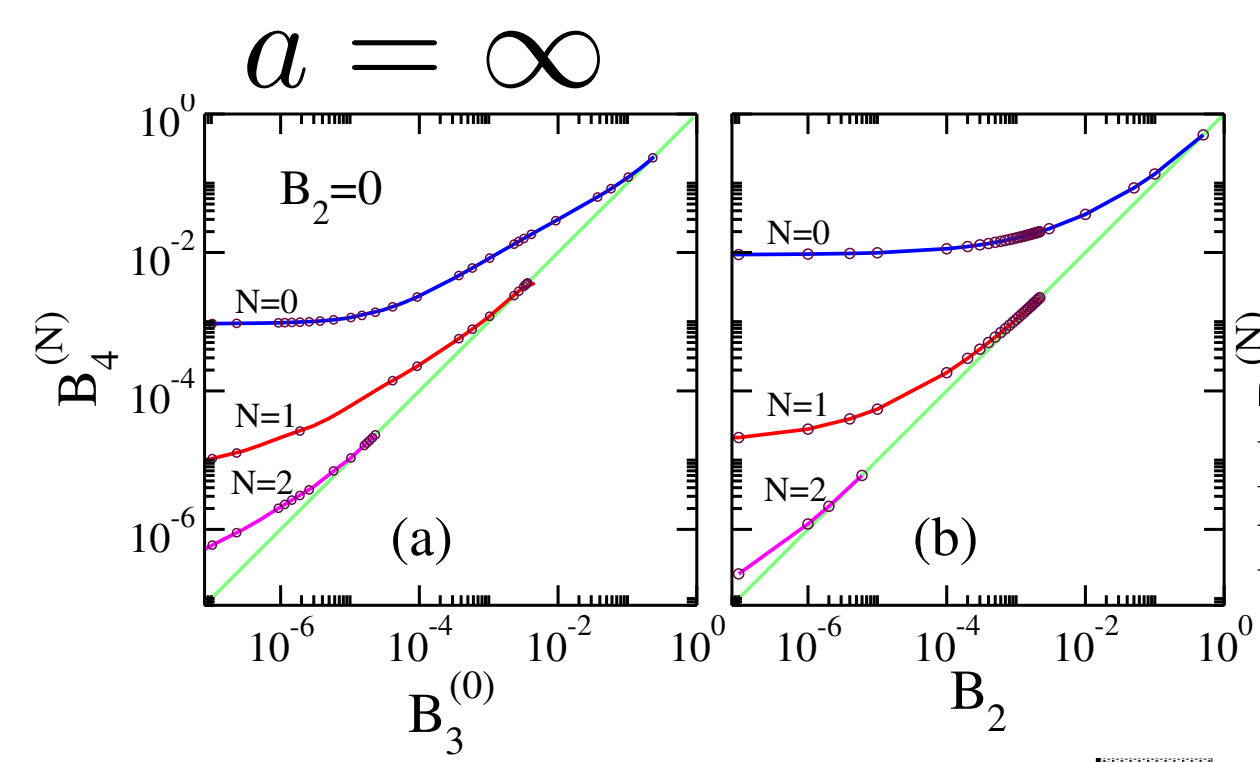

$$
n^{m}
$$

Hadizadeh, Yamashita,Tomio, Delfino, TF, PRL107, 135304 (2011

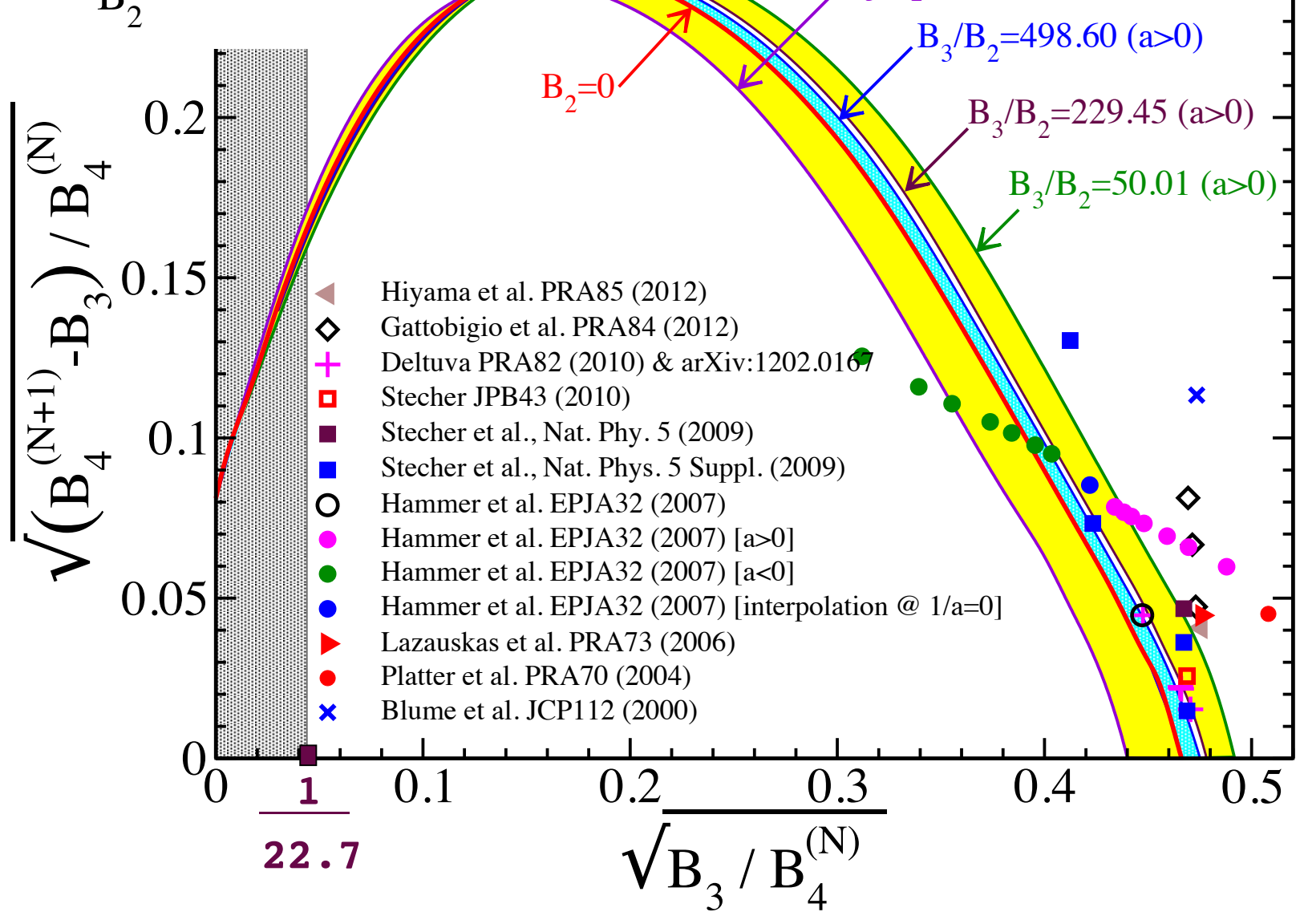


H.W. Hammer and L. Platter, Universal properties of the four-body system with large scattering lengths, European Physical Journal 32, 113 (2007)

- Zero-range potential - EFT

- Three-body repulsive potential to deal with the Thomas collapse

Repulsive interaction at short distances kills the four-body scale.

How to check the 4-boson scale? Use an attractive interaction

J. von Stecher, J.P. D'Incao, C.H. Greene, Signatures of universal four-body phenomena and their relation to the Efimov effect, Nature Physics 5, 417 (2009).

-Two-body Gaussian potential - Stochastic variational method

M. Gattobigio, A. Kievsky, M. Viviani, Energy spectra of small bosonic clusters having a large two-body scattering length, Physical Review A 86, 042513 (2012).

-Two-body Gaussian LM2M2 potentials - Hyperspherical formalism

How to check 4-boson scale? Disentangle the scales by a four-body potential

Experimental verification?

Technique to tune three or four-body

potentials!!!! 


\section{Halo Nuclei and Efimov physics $(n+n+$ core $)$}

Fedorov, Jensen, Riisager, "Efimov states in halo nuclei" PRL73 (1994) $2817 .{ }^{14} \mathrm{Be}{ }^{18} \mathrm{C}{ }^{20} \mathrm{C}$

Mazumdar, Bhasin, "Efimov effect in the nuclear halo 14Be nucleus” PRC 56 (1997) R5

Amorim, TF, Tomio "Universal aspects of Efimov states and light halo nuclei", PRC 56, R2378 (1997)

Mazumdar, Arora, Bhasin, "Three-body analysis of the occurrence of Efimov states in 2n

halo nuclei such as ${ }^{19} \mathrm{~B},{ }^{22} \mathrm{C}$, and ${ }^{20} \mathrm{C} "$, PRC61 (2000) 051303

\section{Halo Nuclei and EFT}

Bertulani, Hammer, van Kolck, "Effective field theory for halo nuclei: shallow p-wave states",

NPA712 (2002) 37

\section{Halo Nuclei, EFT and Efimov physics}

Hammer, Platter, "Efimov States in Nuclear and Particle Physics”, Annu. Rev. Nucl. Part. Sci. 60 (2010) 207 
C. C. Hall, ${ }^{1}$ E. M. Lunderberg, ${ }^{1}$ P. A. DeYoung, ${ }^{1 *}$ T. Baumann, ${ }^{2}$ D. Bazin, ${ }^{2}$ G. Blanchon ${ }^{3}$ A. Bonaccorso, ${ }^{4}$ B. A. Brown, ${ }^{2,5}$ J. Brown, ${ }^{6}$ G. Christian, ${ }^{2,5}$ D. H. Denby, ${ }^{1}$ J. Finck, ${ }^{7}$ N. Frank,${ }^{2,5, \dagger}$ A. Gade, ${ }^{2,5}$ J. Hinnefeld, ${ }^{8}$ C. R. Hoffman,,${ }^{9,10}$ B. Luther, ${ }^{11}$

$$
\text { S. Mosby, }{ }^{2,5} \text { W. A. Peters, }{ }^{2,5, \ddagger} \text { A. Spyrou, }{ }^{2,5} \text { and M. Thoennessen }{ }^{2,5}
$$

The neutron-unbound ground state and two excited states of ${ }^{12} \mathrm{Li}$ were formed by the two-proton removal reaction from a $53.4-\mathrm{MeV} / \mathrm{u}{ }^{14} \mathrm{~B}$ beam. The decay energy spectrum of ${ }^{12} \mathrm{Li}$ was measured with the Modular Neutron Array (MoNA) and the Sweeper dipole superconducting magnet at the National Superconducting Cyclotron Laboratory. Two excited states at resonance energies of $250 \pm 20 \mathrm{keV}$ and $555 \pm 20 \mathrm{keV}$ were observed for the first time and the data are consistent with the previously reported $s$-wave ground state with a scattering length of $a_{s}=-13.7 \mathrm{fm}$

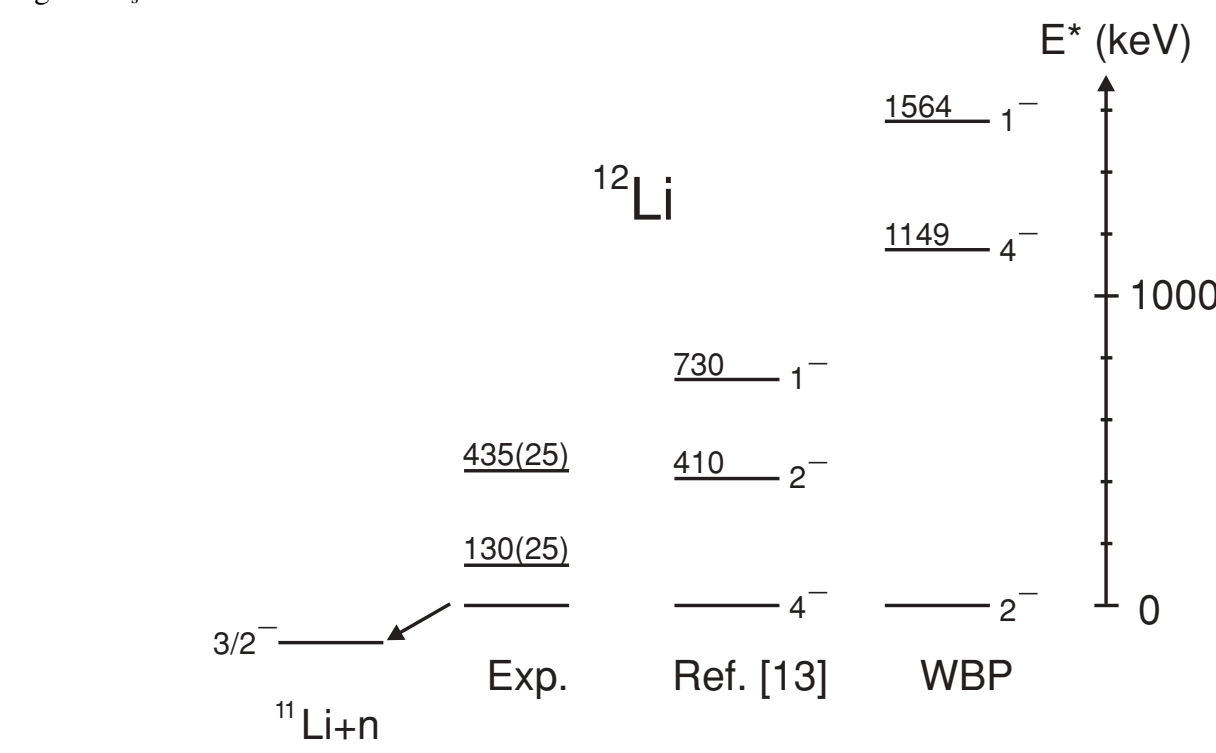

\section{${ }^{12} L i={ }^{9} L i+n+n+n$}

Pauli principle kills sensitivity to the 4-body scale! 
Scales of s-wave n-n-c system: contact interaction

$E_{n n} \quad$ Energy of the virtual nn system

$E_{n c} \quad$ Energy of the bound/virtual nc system

$B_{N}=\left|E_{3}^{(N)}\right| \quad$ Energy of the Nth state of the nnc system

$A=$ mass of the core 


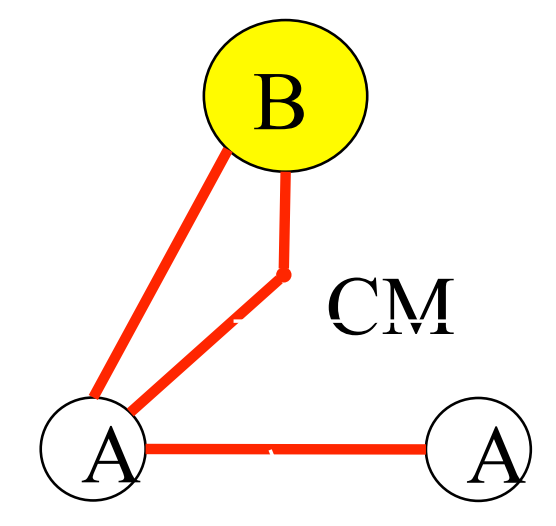

$$
\begin{aligned}
& \sqrt{\left\langle r_{A \gamma}^{2}\right\rangle\left|E_{3}\right|}=R_{A \gamma}\left( \pm \sqrt{\frac{E_{A A}}{E_{3}}}, \pm \sqrt{\frac{E_{A B}}{E_{3}}}, \mathrm{~A}\right) \\
& \sqrt{\left\langle r_{\gamma}^{2}\right\rangle\left|E_{3}\right|}=R_{\gamma}^{C M}\left( \pm \sqrt{\frac{E_{A A}}{E_{3}}}, \pm \sqrt{\frac{E_{A B}}{E_{3}}}, \mathrm{~A}\right) \\
& \begin{array}{ll}
\gamma=A \text { or } B & + \text { two-body bound state } \\
& \text { - two-body virtual state }
\end{array}
\end{aligned}
$$

\section{Build constraints!}
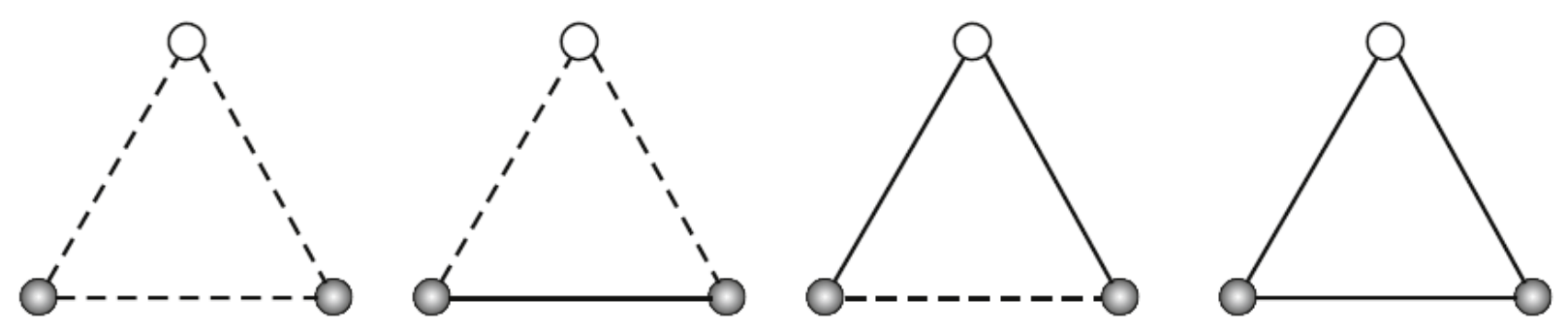

System size for fixed 3-body binding 


\section{Root mean square radii: Core+neutron+neutron}
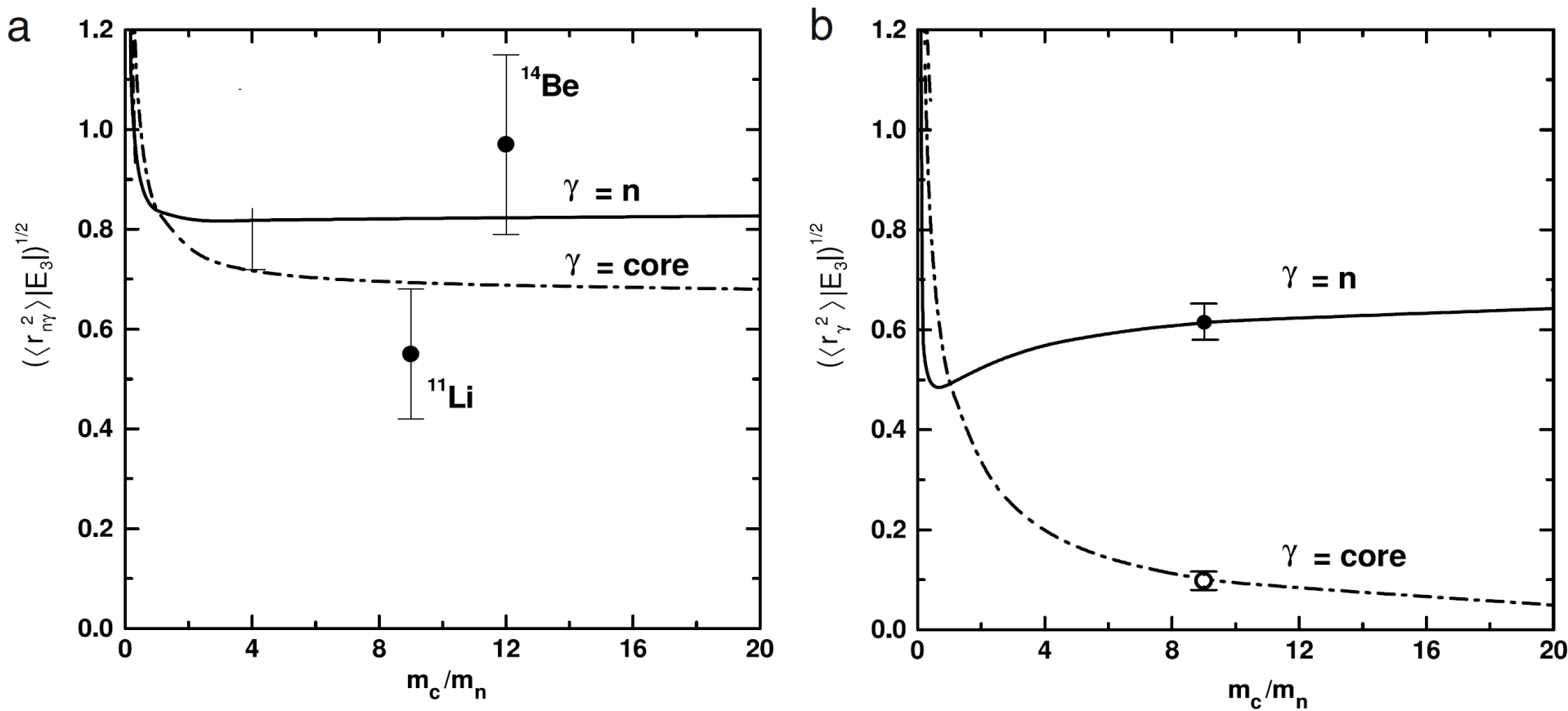

The experimental values of the charge radius of ${ }^{9} \mathrm{Li}$ and ${ }^{11} \mathrm{Li}$ are given in [4] as 2.217(35) and 2.467(37) fm, respectively, such that $\sqrt{\left\langle r_{c h}^{2}\left({ }^{11} \mathrm{Li}\right)\right\rangle-\left\langle\mathrm{r}_{c h}^{2}\left({ }^{9} \mathrm{Li}\right)\right\rangle}=1.08(11) \mathrm{fm}$. A neutron halo radius of $6.54(38) \mathrm{fm}$ was obtained from the extracted matter radius in the experiment performed by [3]. Together with $S_{2 n}=369.15(65) \mathrm{keV}$, reported in [176] for ${ }^{11} \mathrm{Li}$, the experimental value of the root-mean-square distance of ${ }^{9} \mathrm{Li}$ in respect to the center-of-mass of ${ }^{11} \mathrm{Li}\left(\sqrt{\left\langle\mathrm{r}_{c}^{2}\right\rangle}\right)$ in units of $\hbar / \sqrt{m_{n} S_{2 n}}$, is 0.10 (1) and the halo radius $\left(\sqrt{\left\langle r_{n}^{2}\right\rangle}\right)$ in such units is $0.617(36)$, these values should be compared with the theoretical results extracted from Fig. 21, of 0.10 and 0.61 , respectively. The agreement with the experimental supports the model assumptions.

[3] P. Egelhof, et al., Eur. J. Phys. A 15 (2002) 27.

[4] R. Sánchez, et al., Phys. Rev. Lett. 96 (2006) 033002.

[176] M. Smith, et al., Phys. Rev. Lett. 101 (2008) 202501. 


\section{Root mean square radii: Core+neutron+neutron}

- Moriguchi et al. PRC88, 024610 (2013) - Yamashita, Tomio and T. F. RIKEN reaction cross-section $\mathbf{r}_{\mathbf{n}} \sim \mathbf{6 . 1} \mathbf{f m} \quad$ NPA 735, 40 (2004)

- $\quad \mathbf{S}_{2 \mathrm{n}}=\mathbf{3 6 9} \mathbf{k e V}$ Smith et al. PRL101(2008)

Canham and Hammer

NPA 836 (2010) 275

\begin{tabular}{||c|c|c|c||c|c|c|c||}
\hline \hline Nucleus & $B_{3}[\mathrm{keV}]$ & $E_{n c}[\mathrm{keV}]$ & $r_{0}[\mathrm{fm}]$ & $\sqrt{\left\langle r_{n n}^{2}\right\rangle}[\mathrm{fm}]$ & $\sqrt{\left\langle r_{n c}^{2}\right\rangle}[\mathrm{fm}]$ & $\sqrt{\left\langle r_{n}^{2}\right\rangle}[\mathrm{fm}]$ & $\sqrt{\left\langle r_{c}^{2}\right\rangle}[\mathrm{fm}]$ \\
\hline${ }^{11} \mathrm{Li}$ & 247 & -25 & 0.0 & $8.7 \pm 0.7$ & $7.1 \pm 0.5$ & $6.5 \pm 0.5$ & $1.0 \pm 0.1$ \\
& 247 & -25 & 1.4 & $8.80 \pm 0.07$ & $7.21 \pm 0.06$ & $6.51 \pm 0.05$ & $1.040 \pm 0.008$ \\
& 247 & $-800[48]$ & 0.0 & $6.8 \pm 1.8$ & $5.9 \pm 1.5$ & $5.3 \pm 1.4$ & $0.9 \pm 0.2$ \\
& 247 & $-800[48]$ & 1.4 & $6.3 \pm 0.5$ & $5.5 \pm 0.4$ & $4.9 \pm 0.4$ & $0.81 \pm 0.06$ \\
\hline${ }^{14} \mathrm{Be}$ & 1120 & $-200[49]$ & 0.0 & $4.1 \pm 0.5$ & $3.5 \pm 0.5$ & $3.2 \pm 0.4$ & $0.40 \pm 0.05$ \\
& 1120 & $-200[49]$ & 1.4 & $3.86 \pm 0.09$ & $3.29 \pm 0.08$ & $3.02 \pm 0.07$ & $0.384 \pm 0.009$ \\
\hline${ }^{12} \mathrm{Be}$ & 3673 & 503 & 0.0 & $3.0 \pm 0.6$ & $2.5 \pm 0.5$ & $2.3 \pm 0.5$ & $0.32 \pm 0.07$ \\
& 3673 & 503 & 1.4 & $3.3 \pm 0.2$ & $2.7 \pm 0.1$ & $2.5 \pm 0.1$ & $0.35 \pm 0.02$ \\
\hline${ }^{18} \mathrm{C}$ & 4940 & 731 & 0.0 & $2.6 \pm 0.7$ & $2.2 \pm 0.6$ & $2.1 \pm 0.5$ & $0.18 \pm 0.05$ \\
& 4940 & 731 & 1.4 & $2.9 \pm 0.2$ & $2.4 \pm 0.2$ & $2.3 \pm 0.2$ & $0.21 \pm 0.01$ \\
\hline${ }^{20} \mathrm{C}$ & 3506 & $530[45]$ & 0.0 & $3.0 \pm 0.7$ & $2.5 \pm 0.6$ & $2.4 \pm 0.5$ & $0.19 \pm 0.04$ \\
& 3506 & $530[45]$ & 1.4 & $3.38 \pm 0.18$ & $2.75 \pm 0.15$ & $2.60 \pm 0.14$ & $0.21 \pm 0.01$ \\
& 3506 & 162 & 0.0 & $2.8 \pm 0.3$ & $2.4 \pm 0.3$ & $2.3 \pm 0.3$ & $0.19 \pm 0.02$ \\
& 3506 & 162 & 1.4 & $3.03 \pm 0.06$ & $2.53 \pm 0.05$ & $2.39 \pm 0.05$ & $0.198 \pm 0.004$ \\
& 3506 & 60 & 0.0 & $2.8 \pm 0.2$ & $2.3 \pm 0.2$ & $2.2 \pm 0.2$ & $0.18 \pm 0.01$ \\
& 3506 & 60 & 1.4 & $2.84 \pm 0.03$ & $2.41 \pm 0.03$ & $2.28 \pm 0.03$ & $0.192 \pm 0.002$ \\
${ }^{20} \mathrm{C}^{*}$ & $65.0 \pm 6.8$ & 60 & 0.0 & $42 \pm 3$ & $38 \pm 3$ & $41 \pm 3$ & $2.2 \pm 0.2$ \\
${ }^{20} \mathrm{C}^{*}$ & $64.9 \pm 0.7$ & 60 & 1.4 & $43.2 \pm 0.5$ & $38.7 \pm 0.4$ & $42.9 \pm 0.5$ & $2.26 \pm 0.02$ \\
\hline \hline
\end{tabular}


Neutron-neutron correlation function

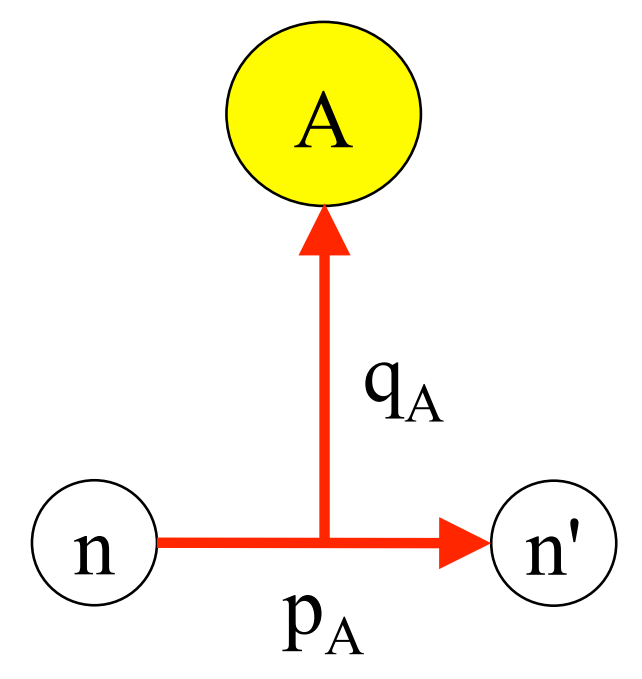

$$
\begin{aligned}
& C_{n n}\left(\vec{p}_{A}\right)=\frac{\int d^{3} q_{A} \mid \Phi\left(\vec{q}_{A}, \vec{p}_{A}\right)^{2}}{\int d^{3} q_{A} \rho\left(\vec{q}_{n}^{\prime}\right) \rho\left(\vec{q}_{n}\right)} \\
& \vec{q}_{n^{\prime}}=\vec{p}_{A}-\frac{\vec{q}_{A}}{2} \quad \vec{q}_{n}=-\vec{p}_{A}-\frac{\vec{q}_{A}}{2}
\end{aligned}
$$

One-body density

$$
\rho\left(\vec{q}_{n A}\right)=\int d^{3} q_{n^{\prime} A}\left|\Phi\left(-\vec{q}_{n A}-\vec{q}_{n^{\prime} A}, \frac{\vec{q}_{n A}-\vec{q}_{n^{\prime} A}}{2}\right)\right|^{2}
$$

$\Phi \equiv \Phi\left(\vec{q}_{A}, \vec{p}_{A}\right) \quad$ Breakup amplitude including the FSI between the neutrons $\Phi=\Psi\left(\vec{q}_{A}, \vec{p}_{A}\right)+\frac{1 /\left(2 \pi^{2}\right)}{\sqrt{E_{n n}}-i p_{A}} \int d^{3} p \frac{\Psi\left(\vec{q}_{A}, \vec{p}\right)}{p_{A}^{2}-p^{2}+i \varepsilon} \quad \Psi$ is the three-body wave function 
Neutron-neutron correlation function

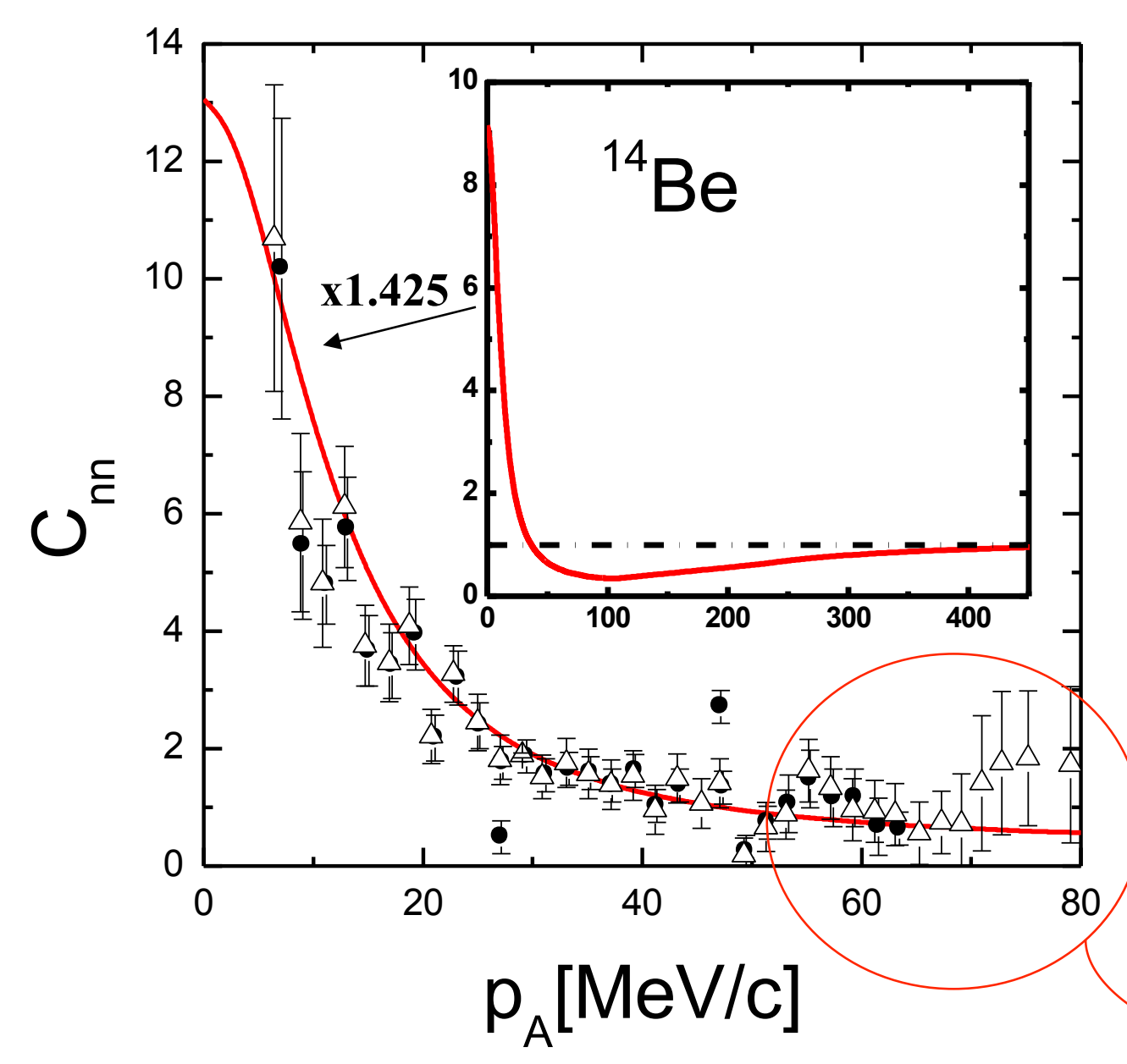

F. M. Marqués et al. Phys. Rev. C 64, 061301 (2001)

F. M. Marqués et al.

Phys. Lett. B 476, 219 (2000)

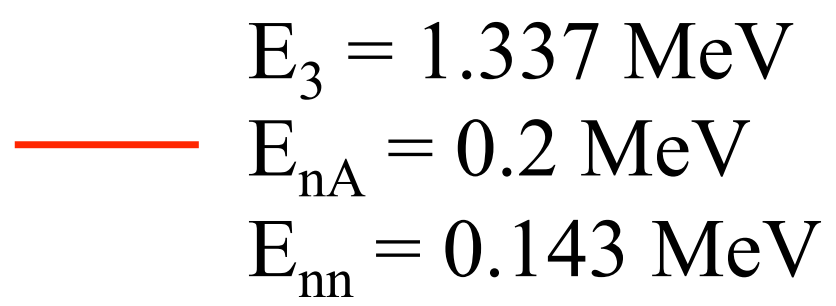

asymptotic region?

Yamashita, TF, Tomio PRC 72, 011601(R) (2005) 


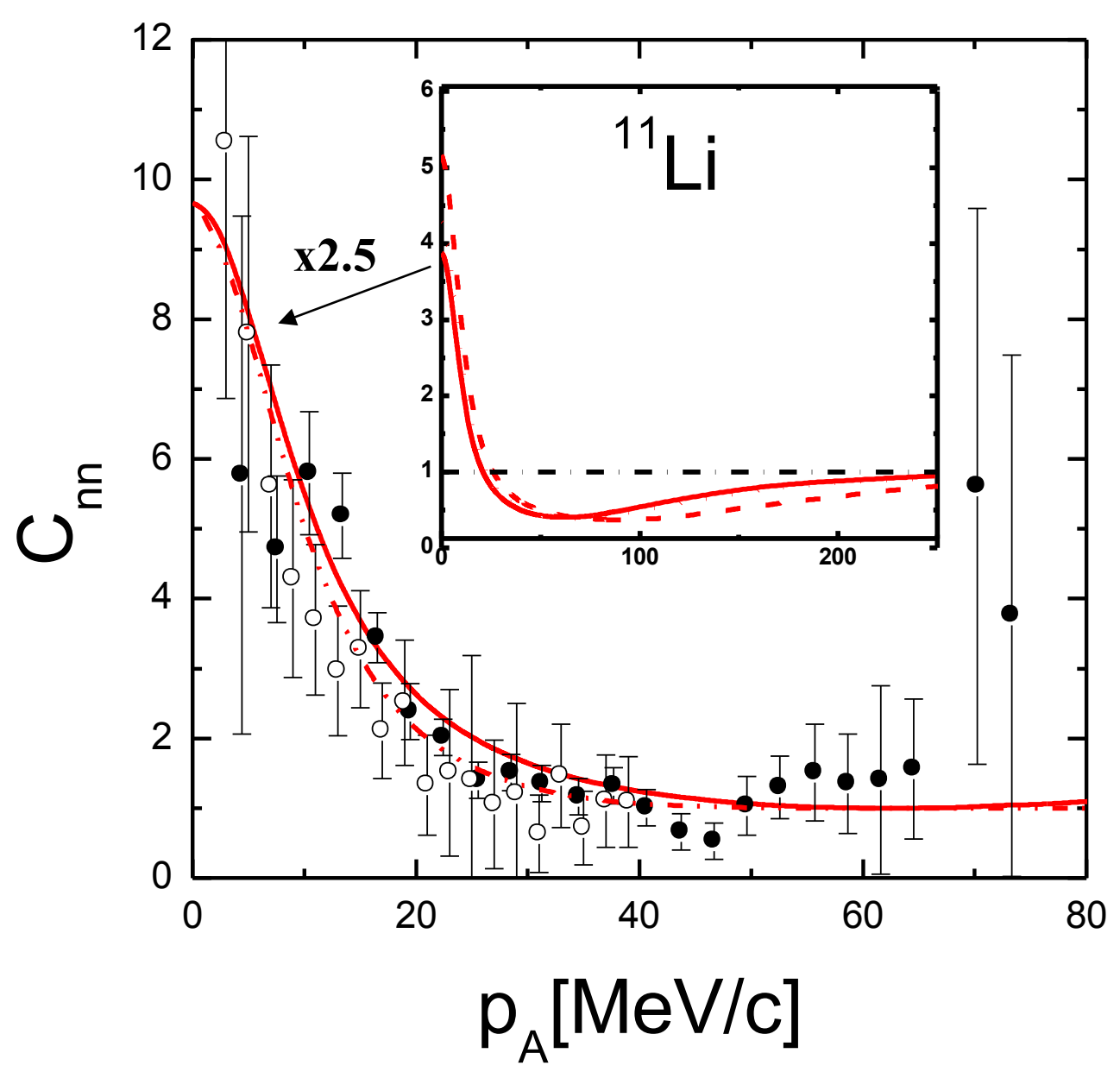

F. M. Marqués et al.

Phys. Rev. C 64, 061301 (2001)

$0-\cdots-$

M. Petrascu et al.

Nucl. Phys. A 738, 503 (2004)

$\begin{array}{ll}\mathrm{E}_{3}=0.29 \mathrm{MeV} \\ \mathrm{E}_{\mathrm{nA}}=0.05 \mathrm{MeV} \\ ---- & \mathrm{E}_{3}=0.37 \mathrm{MeV} \\ & \mathrm{E}_{\mathrm{nA}}=0.8 \mathrm{MeV} \\ & \mathrm{E}_{3}=0.37 \mathrm{MeV} \\ & \mathrm{E}_{\mathrm{nA}}=0.05 \mathrm{MeV}\end{array}$

Enn $=0.143 \mathrm{MeV}$ 


\section{Threshold for an excited Efimov state: Hallo-nuclei}

Critical condition for an excited $(\mathrm{N}+1)$-th above the $\mathrm{N}$-th state

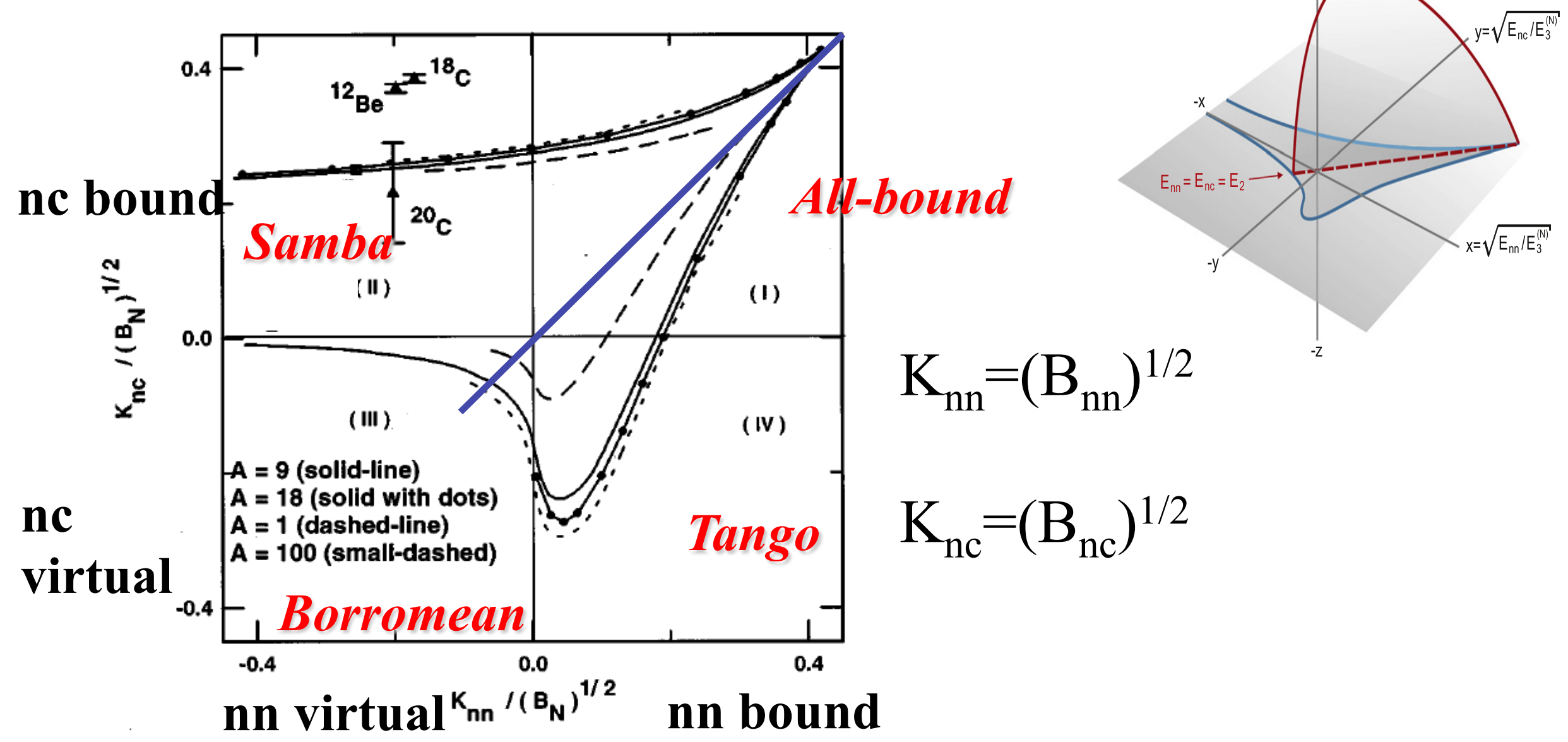

Amorim,TF,Tomio PRC56(1997)2378

Canham and Hammer EPJ A 37 (2008) 367; NPA 836 (2010) 275 


\section{analytic structure \& Efimov state trajectory}

a

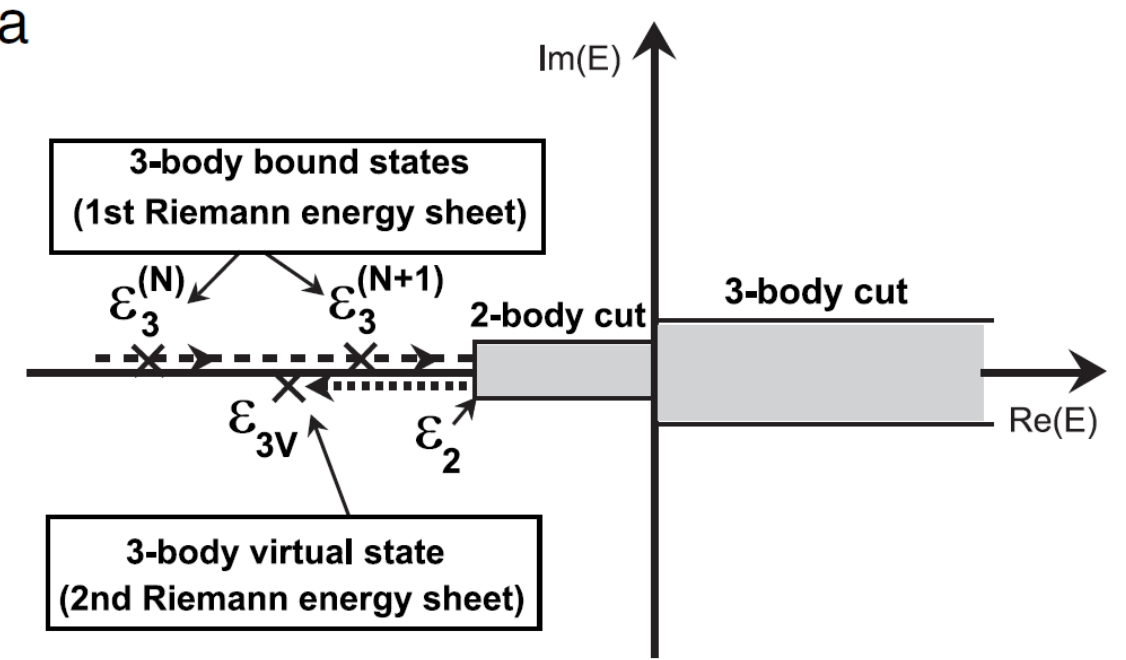

S.K. Adhikari and L. Tomio, Phys. Rev. C 26, 83 (1982); S.K. Adhikari, A.C. Fonseca, and L. Tomio, ibid. 26, 77 (1982). b

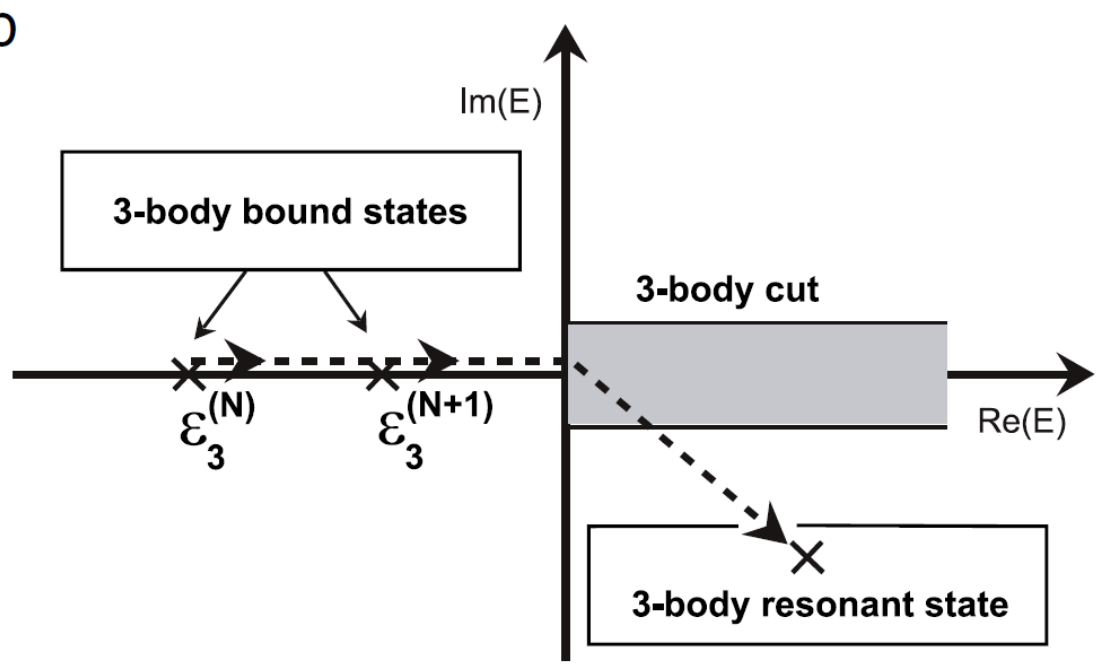

F. Bringas, M.T. Yamashita and T. Frederico, Phys. Rev.A 69, 040702(R) (2004). 


\section{${ }^{20} \mathrm{C}$ virtual Efimov state}

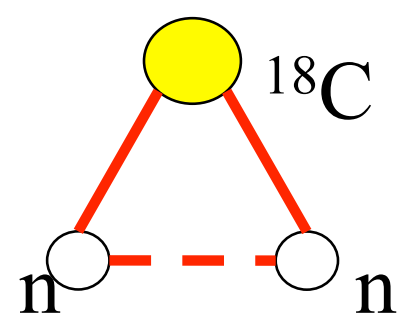

$$
\begin{aligned}
& \mathrm{S}_{2 \mathrm{n}}=3.5 \mathrm{MeV} \\
& \mathrm{E}_{\mathrm{nc}}=160 \pm 110 \mathrm{keV}
\end{aligned}
$$

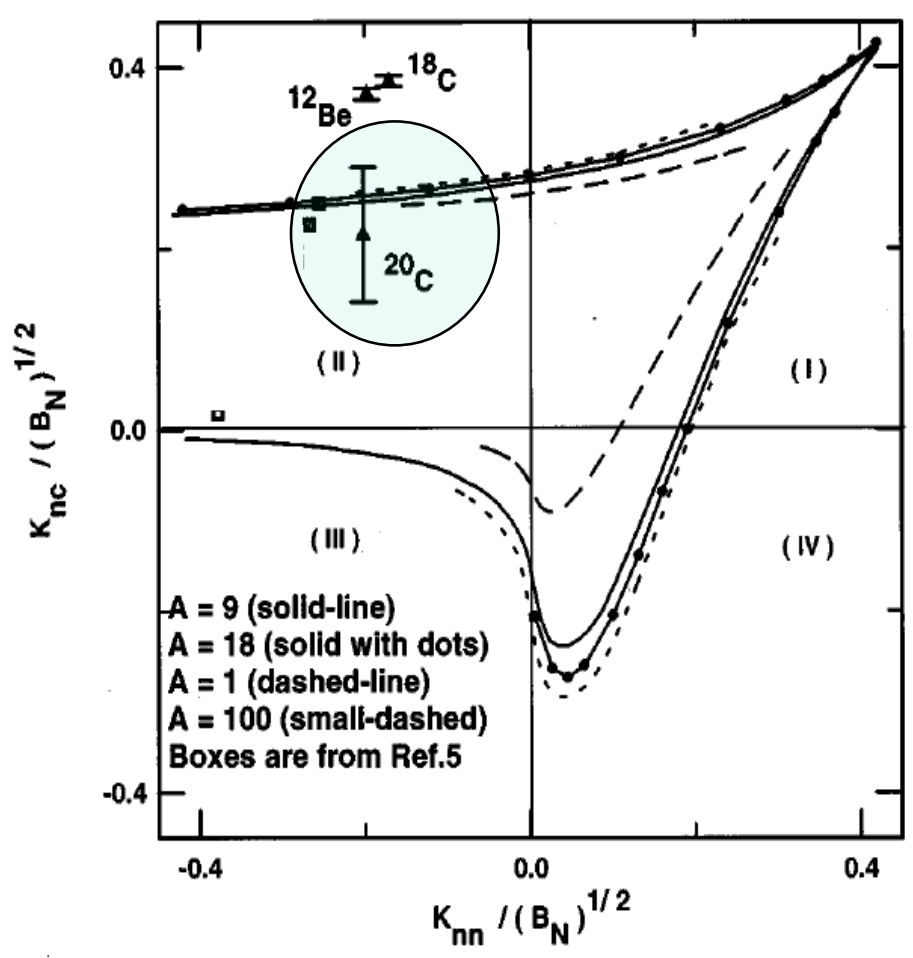

Arora, Mazumdar, Bhasin PRC69 (2004)061301(R) Mazumdar, Rau, Bhasin PRL97(2006)062503 Efimov state $\rightarrow$ Fano resonance of $n+{ }^{19} \mathrm{C}$ by changing $\mathrm{E}_{\mathrm{nc}}$

Yamashita, TF,Tomio, PRL99 (2007)269201 \& PLB660(2008)339

Efimov state $\rightarrow$ virtual state by changing $\mathrm{E}_{\mathrm{nc}}$ 


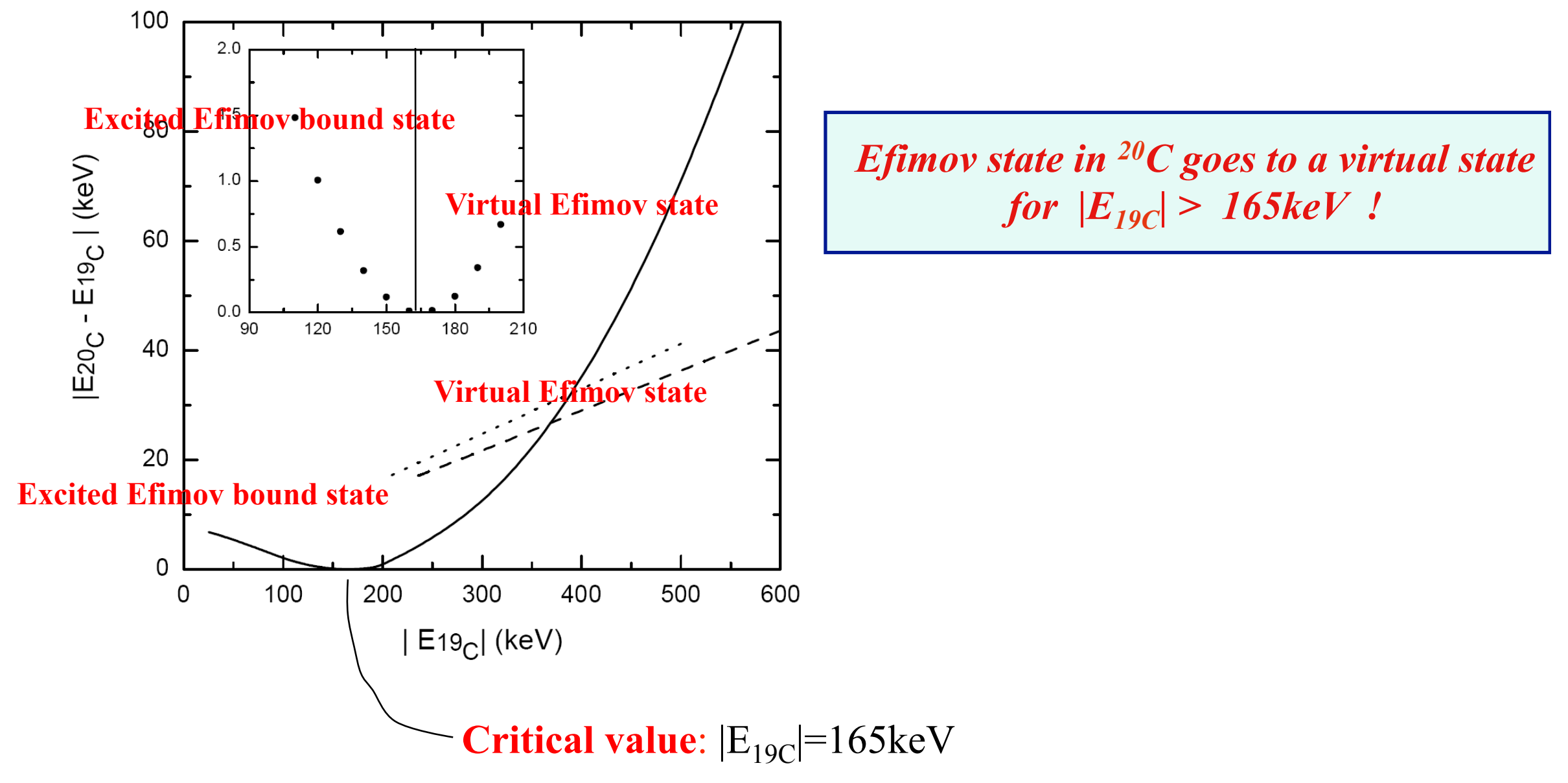

T. Nakamura, et al., Phys. Rev. Lett. 83 (1999) 11112.

$$
S_{n}\left[{ }^{19} C\right]=530 \pm 130 \mathrm{keV}
$$




\section{$n^{-19} \mathrm{C}$ scattering and Efimov physics}

What to expect for s-wave scattering?

Look at doublet neutron-deuteron scattering...

$\Longrightarrow$ Pole in s-wave $k \cot (\delta)$ for $n-d$ system ! Well known $\sim 50$ years

Delves' 60, Van oers \& Seagrave' 67, Girard \& Fuda' 78

$$
k \cot \delta_{0}=-A+B k^{2}-\frac{C}{1+D k^{2}}, \quad \begin{aligned}
& \text { The existence of the triton virtual } \\
& \text { state was found on the basis of the } \\
& \text { effective range expansion. }
\end{aligned}
$$

$\square$ Universal property!

The atom-dimer (three-boson) scattering length is approximately given in Bratten and Hammer (Phys. Rep. 428 (2006) 259):

$$
a_{A D}=\left(1.46-2.15 \tan \left[s_{0} \ln \left(a \Lambda_{*}\right)+0.09\right]\right) a,
$$

where $s_{0}=1.00624$. 


\section{n- ${ }^{19} \mathrm{C}$ scattering and Efimov physics}

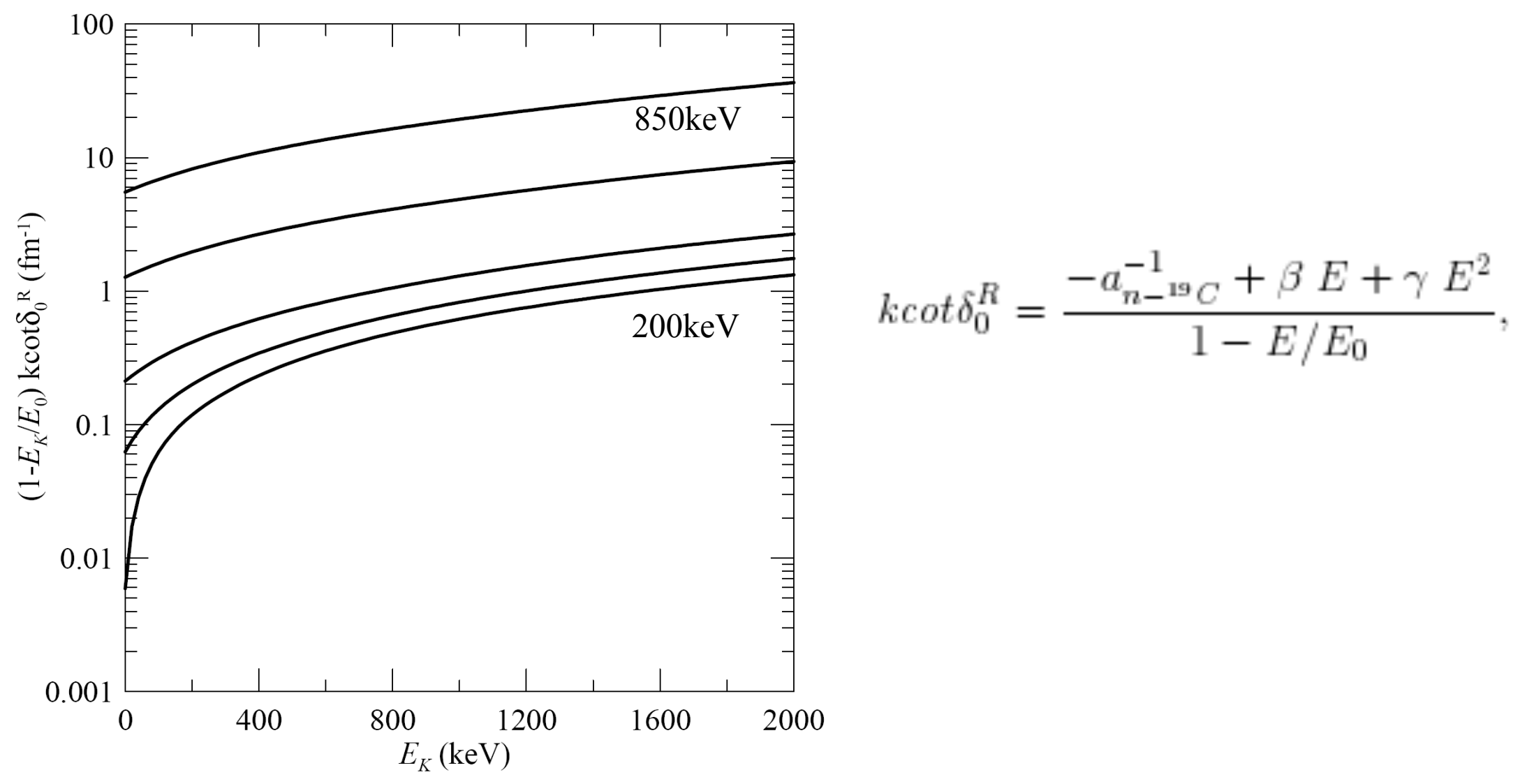

\begin{tabular}{|ccccc|}
\hline$\left|E_{19} \mathrm{C}\right|(\mathrm{keV})$ & $\left(a_{n-19}\right)^{-1}\left(\mathrm{fm}^{-1}\right)$ & $\beta(\mathrm{fm} . \mathrm{keV})^{-1}$ & $\gamma\left(\mathrm{fm} . \mathrm{keV}^{2}\right)^{-1}$ & $E_{0}(\mathrm{keV})$ \\
\hline 200 & $-0.59110^{-2}$ & $5.68510^{-4}$ & $4.67310^{-8}$ & 1442.745 \\
400 & $-0.62410^{-1}$ & $6.74310^{-4}$ & $8.82110^{-8}$ & 823.887 \\
600 & $-2.11810^{-1}$ & $9.33710^{-4}$ & $1.46410^{-7}$ & 451.398 \\
800 & -1.268 & $3.1110^{-3}$ & $4.42410^{-7}$ & 114.976 \\
850 & -5.510 & $1.20110^{-2}$ & $1.64110^{-6}$ & 28.845 \\
\hline
\end{tabular}




\section{$n-{ }^{19} \mathrm{C}$ scattering and Efimov physics}

${ }^{20} \mathrm{C}$ has a virtual Efimov state

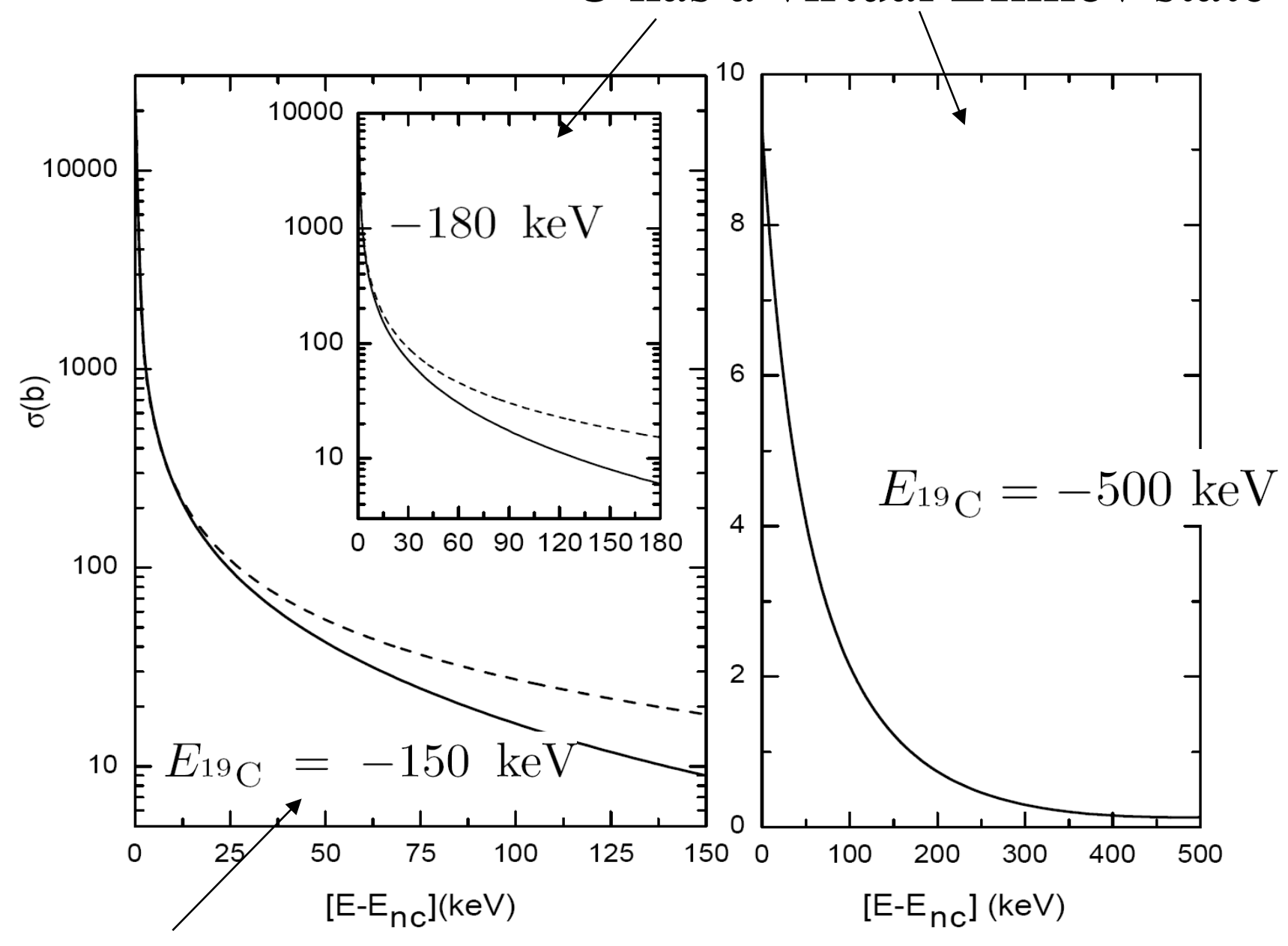

${ }^{20} \mathrm{C}$ has an excited bound Efimov state 


\section{${ }^{22} \mathrm{C}=n-n={ }^{20} \mathrm{C}$}

K. Tanaka et al., Phys. Rev. Lett. 104 (2010) 062701

Reaction cross sections $\left(\sigma_{R}\right)$ for ${ }^{19} \mathrm{C},{ }^{20} \mathrm{C}$ and the drip-line nucleus ${ }^{22} \mathrm{C}$ on a liquid hydrogen target have been measured at around $40 \mathrm{~A} \mathrm{MeV}$ by a transmission method. A large enhancement of $\sigma_{R}$ for ${ }^{22} \mathrm{C}$ compared to those for neighboring $\mathrm{C}$ isotopes was observed. Using a finite-range Glauber calculation under an optical-limit approximation the rms matter radius of ${ }^{22} \mathrm{C}$ was deduced to be $5.4 \pm 0.9 \mathrm{fm}$. It does not follow the systematic behavior of radii in carbon isotopes with $N \leq 14$, suggesting a neutron halo. It was found by an analysis based on a few-body Glauber calculation that the two-valence neutrons in ${ }^{22} \mathrm{C}$ preferentially occupy the $1 s_{1 / 2}$ orbital.

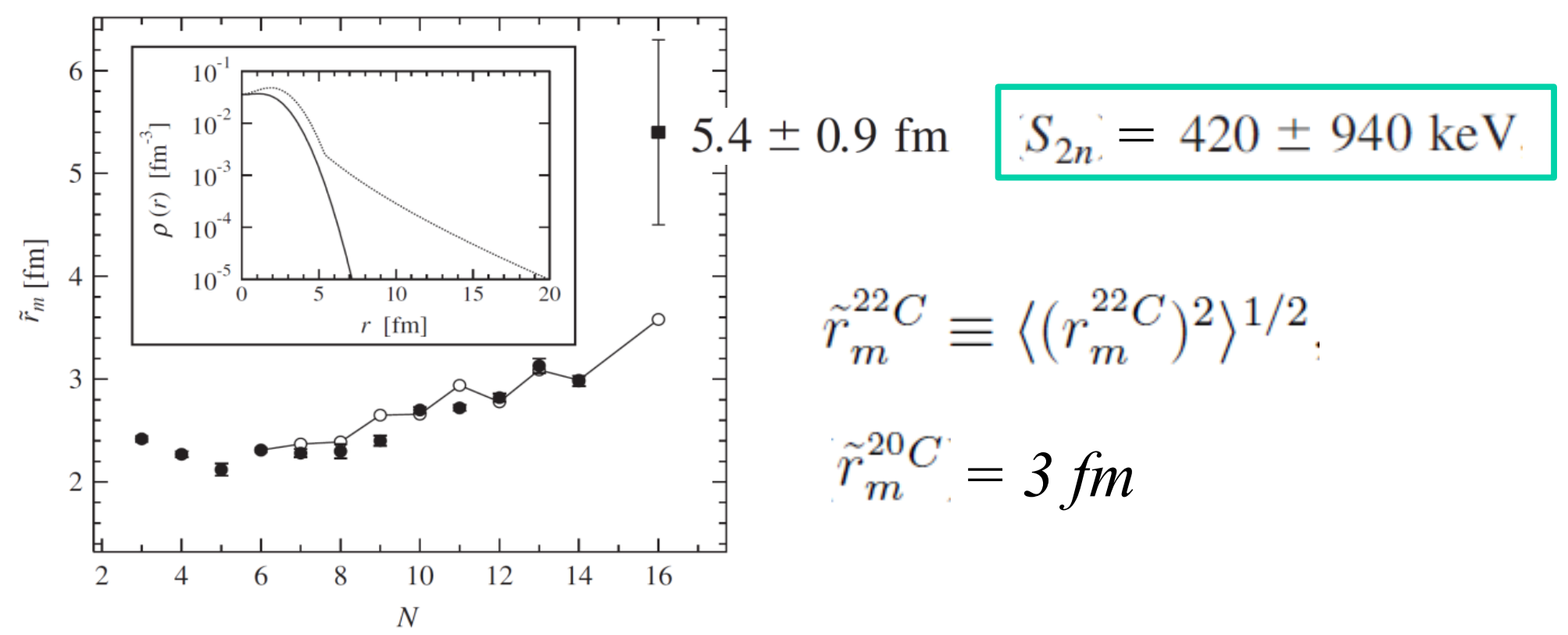

$$
\tilde{r}_{n}^{22 C}=\sqrt{\frac{22}{2}} \sqrt{\left(\tilde{r}_{m}^{22} C\right)^{2}-\frac{20}{22}\left(\tilde{r}_{m}^{20} C\right)^{2}} \approx 15 \pm 3 f m
$$


${ }^{22} C=n-n-{ }^{20} C$

${ }^{21} \mathrm{C}$ virtual state energy 0, $-100 \mathrm{KeV} . E_{n n}=-143 \mathrm{KeV}$

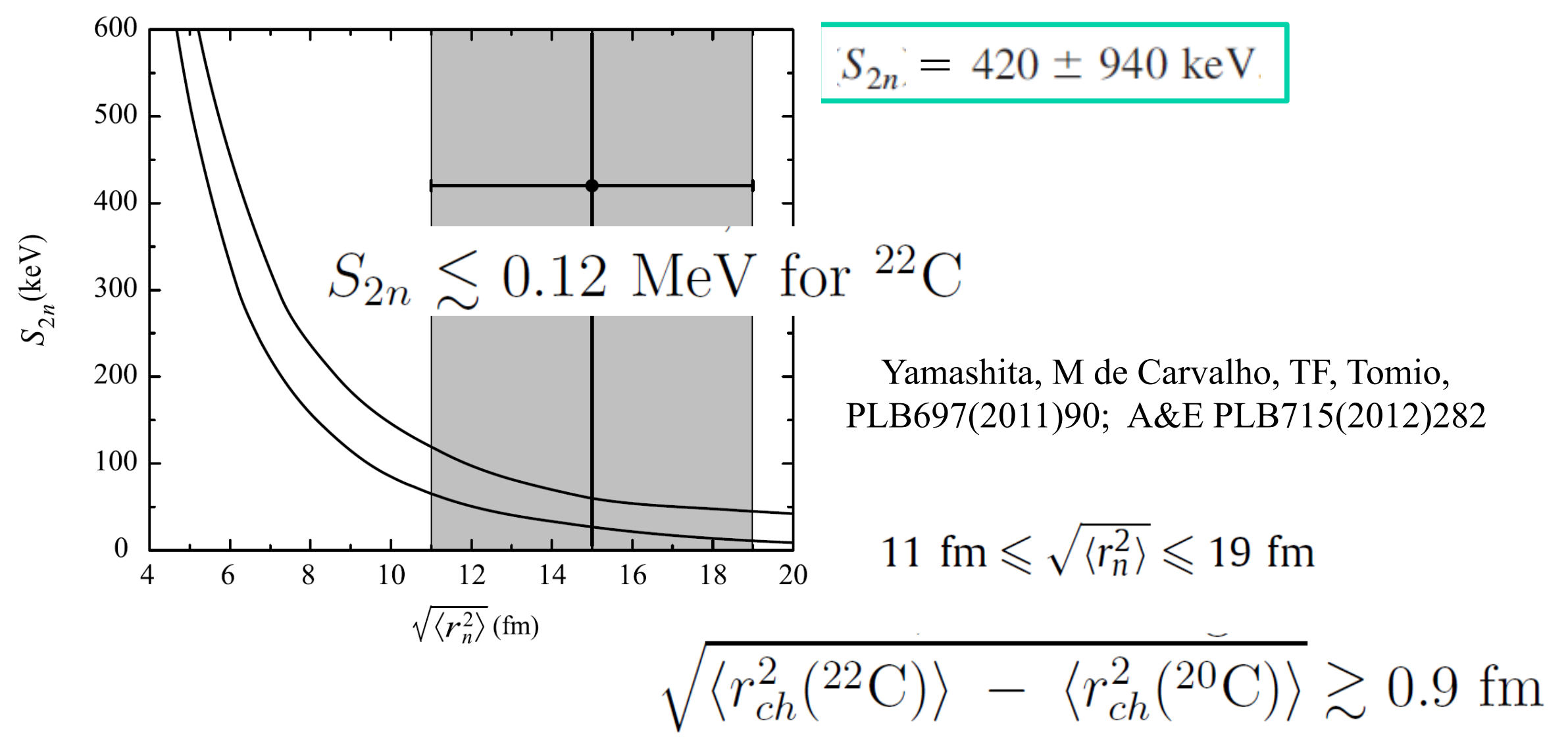

H.T. Fortune, R. Sherr, Phys. Rev. C 85 (2012) 027303.

Acharya, Ji, Phillips PLB723(2013)19 $\left[\mathrm{S}_{2 \mathrm{n}}<100 \mathrm{keV}\right]($ EFT)

Horiuchi and Y. Suzuki, Phys. Rev. C 74, 034311 (2006) 
${ }^{22} C=n-n={ }^{20} C$

${ }^{21} \mathrm{C}$ Mosby et al. NPA 909, 69 (2013) - MSU - $\left|\mathrm{a}_{\mathrm{s}}\right|<2.8 \mathrm{fm}\left({ }^{21} \mathrm{C}\right.$ virtual state $)$

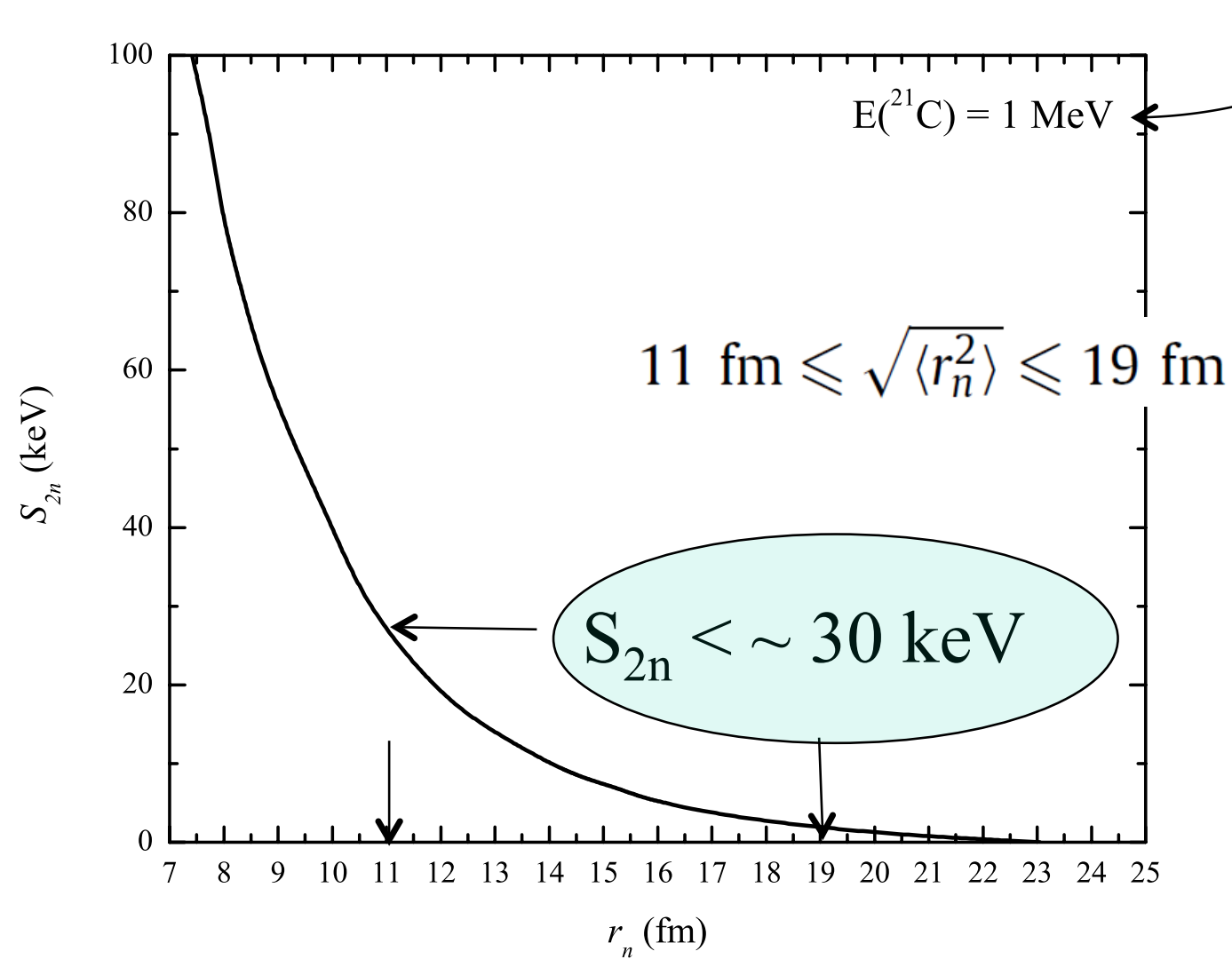




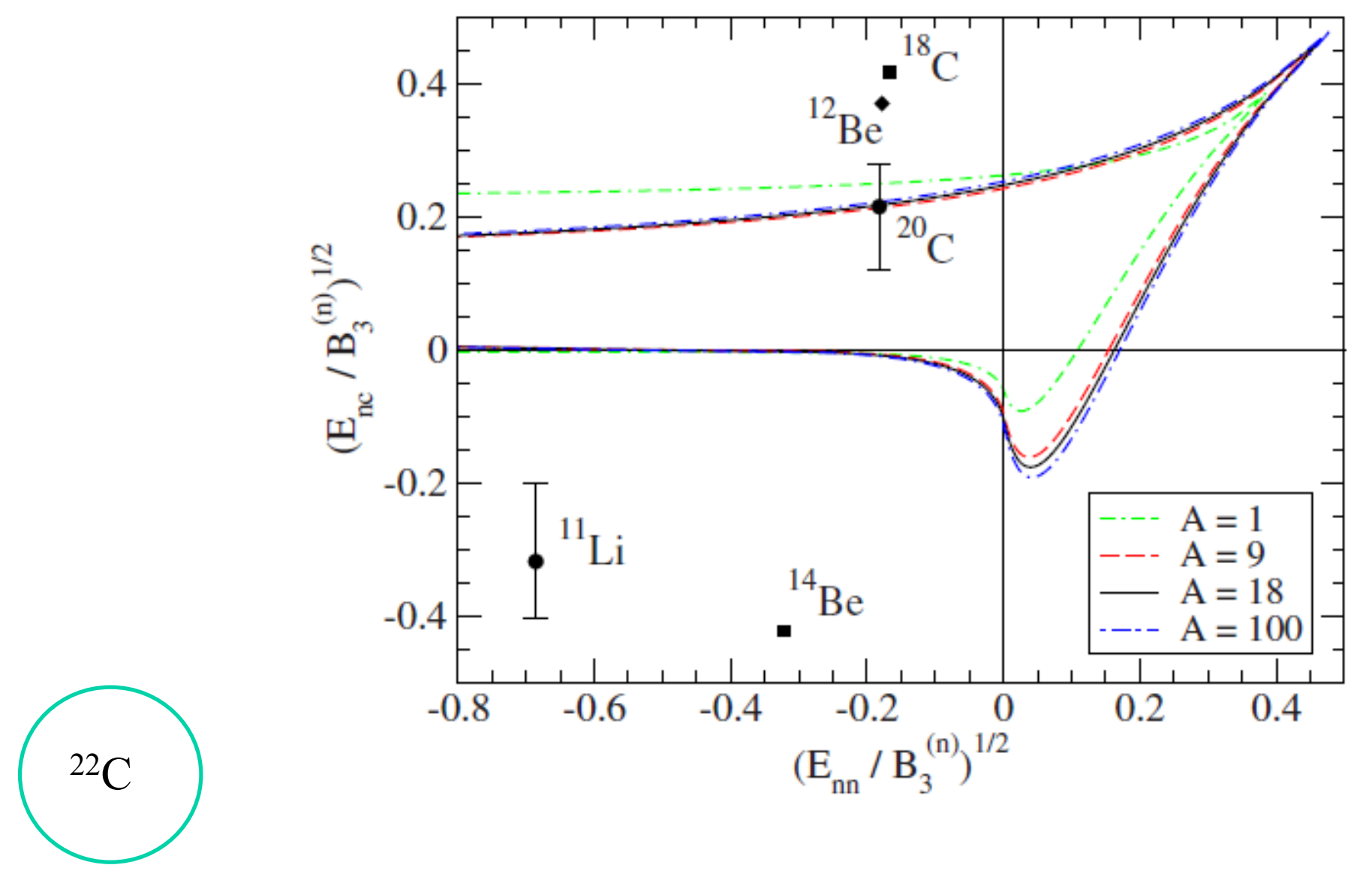

${ }^{21} \mathrm{C}$ with a virtual state with energy $1 \mathrm{MeV}$

$\rightarrow$ It is not possible an excited Efimov state/continuum resonance 
Limit cycle: one-body momentum densities $n+n+A(A=20)$

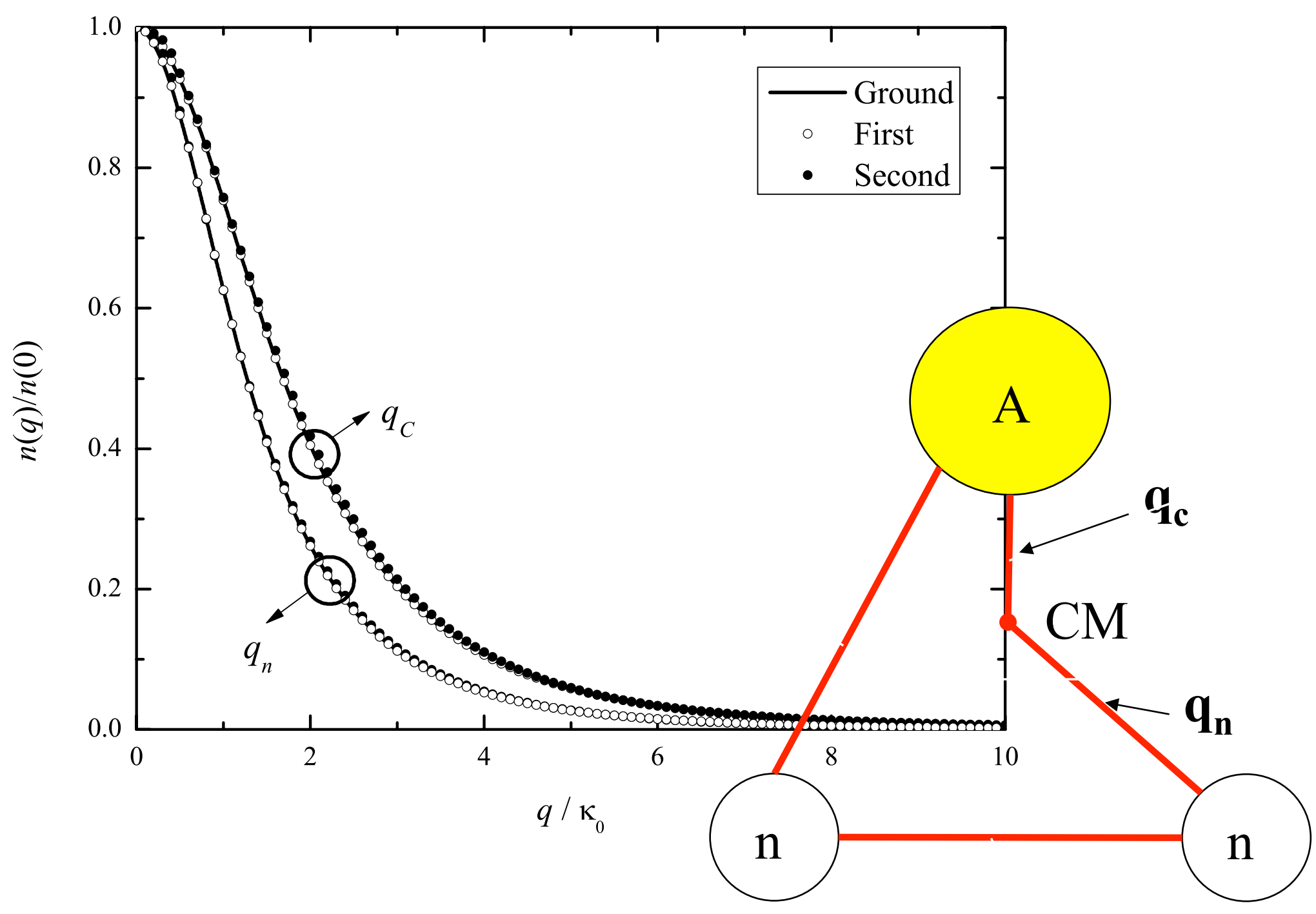

Halo-neutron momentum distribution in ${ }^{22} \mathrm{C}$ 


\section{If $\mathrm{L}_{\text {total }}$ is nonzero ?}

- Virtual p-wave states of light non Borromean nn halo nuclei

$$
\mathrm{E}_{\text {virtual }} \sim 1.7 \mathrm{E}_{\mathrm{nc}}
$$

(Delfino et al PRC61, 051301 (2000))

- Soft dipole mode:

- M. Cubero et al, PRL 109, 262701 (2012) $-{ }^{11} \mathrm{Li}+208 \mathrm{~Pb}$ close the Coulomb barrier $\rightarrow \mathrm{E}_{\text {res }}=690 \mathrm{keV}$ width $=0.32 \mathrm{keV}$

- Fernandez-Garcia et al PRL 110, 142701 (2013) - ${ }^{11} \mathrm{Li}+{ }^{208} \mathrm{~Pb}$ breakup around the Coulomb barrier

- Ershov, Vaagen, Zhukov, PRC 86 (2012) $034331-{ }^{22} \mathrm{C}$

Determined by scattering lenghts only! 


\section{Summary}

$\Longrightarrow$ Weakly bound \& large systems: few scales regime in halo nuclei, molecules, trapped atoms CORRELATIONS BETWEEN OBSERVABLES $\rightarrow$ CONSTRAINTS!

$\Longrightarrow$ Zero-range model n-n-c system:

threshold conditions for excited states and resonances borromean configuration: Efimov state $\rightarrow$ resonance at least one subsystem is bound: Efimov state $\rightarrow$ virtual state

$\Longrightarrow$ Few-examples: ${ }^{11} \mathrm{Li},{ }^{14} \mathrm{Be},{ }^{20} \mathrm{C},{ }^{22} \mathrm{C}$

$\Rightarrow{ }^{20} \mathrm{C}$ Efimov state $\rightarrow$ virtual state $\mathrm{E}_{19 \mathrm{C}}>165 \mathrm{keV}$

$\Longrightarrow{ }^{22} \mathrm{C}$ large $\mathrm{nn}$ halo $\mathrm{S}_{2 \mathrm{n}} \sim 30 \mathrm{keV}$ with ${ }^{21} \mathrm{C}$ virtual state $1 \mathrm{MeV}\left(\right.$ from $\left.\left|\mathrm{a}_{\mathrm{s}}\right|<2.8 \mathrm{fm}\right) \rightarrow$ No Efimov continuum resonance/excited state (range corrections?) 


\section{Outlook}

$\Longrightarrow$ Neutron halo $>2 \mathrm{n}$ (no need of a 4-body scale)...

$\Longrightarrow{ }^{12} \mathrm{Li}={ }^{10} \mathrm{Li}+\mathrm{n}+\mathrm{n}+\mathrm{n},{ }^{21} \mathrm{C}={ }^{18} \mathrm{C}+\mathrm{n}+\mathrm{n}+\mathrm{n}$

$\square$ Exploration of universality in scattering, breakup of halo nuclei \& CDCC ...

$\longrightarrow$ Pigmy resonances $\mathrm{L}_{\text {total }}=1,2,3 \ldots$

$\square \quad$ Fix the tail of ab-initio calculations... 
Collaborators:

\author{
Antonio Delfino (UFF/Brazil) \\ Filipe Bellotti (PhD/ITA/Aarhus) \\ Mohammadreza Hadizadeh (Ohio Univ) \\ Lauro Tomio (IFT/Brazil) \\ Marcelo Yamashita (IFT/Brazil)
}

